\title{
Mean Square Error for the Leland-Lott Hedging Strategy: Convex Pay-Offs
}

Received: date / Accepted: date

\begin{abstract}
The Leland strategy of approximate hedging of the call-option under proportional transaction costs prescribes to use, at equidistant instants of portfolio revisions, the classical Black-Scholes formula but with a suitably enlarged volatility. An appropriate mathematical framework is a scheme of series, i.e. a sequence of models $M_{n}$ with the transaction costs coefficients $k_{n}$ depending on $n$, the number of the revision intervals. The enlarged volatility $\widehat{\sigma}_{n}$, in general, also depends on $n$. Lott investigated in detail the particular case where the transaction costs coefficients decrease as $n^{-1 / 2}$ and where the Leland formula yields $\widehat{\sigma}_{n}$ not depending on $n$. He proved that the terminal value of the portfolio converges in probability to the pay-off $g\left(S_{T}\right)$ where $G(x)=(x-K)^{+}$. In the present note we consider the case of much more general convex piecewise smooth pay-off functions $G$. We show that the convergence holds also in $L^{2}$ and find the first order term of asymptotics for the mean square error. We are working in the setting with non-uniform revision intervals and establish the asymptotic expansion when the revision dates are $t_{i}^{n}=g(i / n)$ where the strictly increasing scale function $g:[0,1] \rightarrow[0,1]$ and its inverse $f$ are continuous with their first and second derivatives on the whole interval or $g(t)=1-(1-t)^{\beta}, \beta \geq 1$.
\end{abstract}

Keywords Black-Scholes formula - Transaction costs · Leland-Lott strategy · Approximate hedging.

E. Denis

Laboratoire de Mathématiques, Université de Franche-Comté, 16 Route de Gray, 25030 Besançon, cedex, France

and School of Management, Boston University, 505 Commonwealth Ave., Boston, MA 02215

E-mail: emmanuel.denis6@wanadoo.fr

Y. Kabanov

Laboratoire de Mathématiques, Université de Franche-Comté, 16 Route de Gray, 25030 Besançon, cedex, France, and Central Economics

and Mathematics Institute, Moscow, Russia

E-mail: Youri.Kabanov@univ-fcomte.fr 
Mathematics Subject Classification (2000) 60G44

JEL Classification G11 · G13

\section{Introduction}

1. Formulation of the main result. To fix the notation we consider the classical Black-Scholes model, already under the martingale measure and with the maturity $T=1$. So, let $S=\left(S_{t}\right), t \in[0,1]$, be a geometric Brownian motion given by the formula

$$
S_{t}=S_{0} e^{\sigma W_{t}-\frac{1}{2} \sigma^{2} t}
$$

and satisfying the linear equation

$$
d S_{t}=\sigma S_{t} d W_{t}
$$

with a standard Wiener process $W$ and a strictly positive constants $S_{0}, \sigma$. The problem is to hedge an option with the pay-off $G\left(S_{1}\right)$ where $G$ is a continuous (or even Borel) function of polynomial growth. Its solution is the following. Let

$$
C(t, x)=E G\left(x e^{\xi \sigma \sqrt{1-t}-\frac{1}{2} \sigma^{2}(1-t)}\right), \quad t \in[0,1], x>0,
$$

where $\xi \sim N(0,1)$. Then $C(t, x)=C(t, x, \sigma)$ is the solution, in the domain $[0,1] \times] 0, \infty[$, of the Cauchy problem

$$
C_{t}(t, x)+\frac{1}{2} \sigma^{2} x^{2} C_{x x}(t, x)=0, \quad C(1, x)=G(x) .
$$

In the particular case, where $G(x)=(x-K)^{+}, K>0$, the function $C(t, x)$ admits an explicit expression and this is the famous Black-Scholes formula:

$$
C(t, x)=C(t, x, \sigma)=x \Phi(d)-K \Phi(d-\sigma \sqrt{1-t}), \quad t<1,
$$

where $\Phi$ is the Gaussian distribution function with the density $\varphi$,

$$
d=d(t, x)=d(t, x, \sigma)=\frac{1}{\sigma \sqrt{1-t}} \ln \frac{x}{K}+\frac{1}{2} \sigma \sqrt{1-t} .
$$

Define the process

$$
V_{t}=C\left(0, S_{0}\right)+\int_{0}^{t} C_{x}\left(u, S_{u}\right) d S_{u} .
$$

In the Ito formula for $C\left(t, S_{t}\right)$ the integral over $d t$ vanishes and, therefore, $V_{t}=C\left(t, S_{t}\right)$ for all $t \in[0,1]$. In particular, $V_{1}=G\left(S_{1}\right)$ : at maturity the value process $V$ replicates the terminal pay-off of the option.

Modelling assumptions of the above formulation are, between others: frictionless market and continuous trading. The latter is a purely theoretical 
invention. Practically, an investor revises the portfolio at certain dates $t_{i}$ and keeps $C_{x}\left(t_{i}, S_{t_{i}}\right)$ units of the stock until the next revision date $t_{i+1}$. The model becomes more realistic if the transactions are charged proportionally to their volume. The portfolio strategy suggested by Leland [6] for asymptotic hedging of the call option generates the value process

$$
V_{t}^{n}=\widehat{C}\left(0, S_{0}\right)+\int_{0}^{t} \sum_{i=1}^{n} H_{t_{i-1}}^{n} I_{\left[t_{i-1}, t_{i}\right]}(u) d S_{u}-\sum_{t_{i}<t} k_{n} S_{t_{i}}\left|H_{t_{i}}^{n}-H_{t_{i-1}}^{n}\right|,
$$

where $H_{t_{i}}^{n}=\widehat{C}_{x}\left(t_{i}, S_{t_{i}}\right), t_{i}=i / n$, the positive parameter $k_{n}=k_{0} n^{-1 / 2}$ is the transaction costs coefficient, and $\widehat{C}(t, x)$ is the solution of (1.1) with $\sigma$ replaced by $\widehat{\sigma}>0$ such that

$$
\widehat{\sigma}^{2}=\sigma^{2}+\sigma k_{0} \sqrt{8 / \pi} .
$$

That is $\widehat{C}(t, x)=C(t, x, \widehat{\sigma})$ and for such a strategy there is no need in a new software: traders can use their old one, changing only one input parameter, the volatility.

In his paper Leland studied the call option and claimed, without providing arguments, that $V_{1}^{n}$ converges to $V_{1}=\left(S_{1}-K\right)^{+}$in probability as $n \rightarrow \infty$. This assertion was proven by Lott in his thesis [8] and we believe that the result could be referred to as the Leland-Lott theorem. In fact, $V_{1}^{n}$ converges also in $L^{2}$ and the following statement gives the rate of convergence:

Theorem 1.1 The mean square approximation error of the Leland-Lott strategy for hedging the European call option with equidistant revision dates has the following asymptotics:

$$
E\left(V_{1}^{n}-V_{1}\right)^{2}=A_{1} n^{-1}+o\left(n^{-1}\right), \quad n \rightarrow \infty,
$$

where the coefficient

$$
A_{1}=\int_{0}^{1}\left[\frac{\sigma^{4}}{2}+\sigma^{3} k_{0} \sqrt{\frac{2}{\pi}}+k_{0}^{2} \sigma^{2}\left(1-\frac{2}{\pi}\right)\right] \Lambda_{t} d t
$$

with $\Lambda_{t}=E S_{t}^{4} \widehat{C}_{x x}^{2}\left(t, S_{t}\right)$. Explicitly,

$$
\Lambda_{t}=\frac{K^{2}}{2 \pi \widehat{\sigma} \sqrt{1-t} \sqrt{2 \sigma^{2} t+\widehat{\sigma}^{2}(1-t)}} \exp \left\{-\frac{\left(\ln \frac{S_{0}}{K}-\frac{1}{2} \sigma^{2} t-\frac{1}{2} \widehat{\sigma}^{2}(1-t)\right)^{2}}{2 \sigma^{2} t+\widehat{\sigma}^{2}(1-t)}\right\} \text {. }
$$

Following [3] we consider a slightly more general hedging strategy with a non-uniform revision grid defined by a smooth transformation of the uniform one.

Let $f$ be a strictly increasing differentiable function on $[0,1]$ such that $f(0)=0, f(1)=1$ and let $g:=f^{-1}$ denote its inverse. For each fixed $n$ we define the revision dates $t_{i}=t_{i}^{n}=g(i / n), 1, \ldots, n$. The enlarged volatility now depends on $t$ and is given by the formula

$$
\widehat{\sigma}_{t}^{2}=\sigma^{2}+\sigma k_{0} \sqrt{8 / \pi} \sqrt{f^{\prime}(t)} .
$$


The pricing function

$$
\widehat{C}(t, x)=E\left(x e^{\xi \rho_{t}-\frac{1}{2} \rho_{t}^{2}}-K\right)^{+}, \quad t \in[0,1], x>0,
$$

where

$$
\rho_{t}^{2}=\int_{t}^{1} \widehat{\sigma}_{s}^{2} d s
$$

admits the explicit expression

$$
\widehat{C}(t, x)=x \Phi\left(\rho_{t}^{-1} \ln (x / K)+\rho_{t} / 2\right)-K \Phi\left(\rho_{t}^{-1} \ln (x / K)-\rho_{t} / 2\right), \quad t<1 .
$$

The function $\rho_{t}$ decreases from $\rho_{0}$ to 0 . The following bounds are obvious:

$$
\sigma^{2}(1-t) \leq \rho_{t}^{2} \leq \sigma^{2}(1-t)+\sigma k_{0} \sqrt{8 / \pi}(1-t)^{1 / 2}(1-f(t))^{1 / 2} .
$$

Assumption 1: $g, f \in C^{2}([0,1])$.

Assumption 2: $g(t)=1-(1-t)^{\beta}, \beta \geq 1$.

Note that in the second case where $f(t)=1-(1-t)^{1 / \beta}$ the derivative $f^{\prime}$ for $\beta>1$ explodes at the maturity date and so does the enlarged volatility. The notation $C^{2}([0,1])$ is used for the function which are continuous with their two derivatives on the closed interval $[0,1]$.

Theorem 1.2 Under any of the above assumptions the mean square approximation error for hedging the European call option has the following asymptotics:

$$
E\left(V_{1}^{n}-V_{1}\right)^{2}=A_{1}(f) n^{-1}+o\left(n^{-1}\right), \quad n \rightarrow \infty,
$$

where the coefficient

$$
A_{1}(f)=\int_{0}^{1}\left[\frac{\sigma^{4}}{2} \frac{1}{f^{\prime}(t)}+k_{0} \sigma^{3} \sqrt{\frac{2}{\pi}} \frac{1}{\sqrt{f^{\prime}(t)}}+k_{0}^{2} \sigma^{2}\left(1-\frac{2}{\pi}\right)\right] \Lambda_{t} d t
$$

with $\Lambda_{t}=E S_{t}^{4} \widehat{C}_{x x}^{2}\left(t, S_{t}\right)$. Explicitly,

$$
\Lambda_{t}=\frac{1}{2 \pi \rho_{t}} \frac{K^{2}}{\sqrt{2 \sigma^{2} t+\rho_{t}^{2}}} \exp \left\{-\frac{\left(\ln \frac{S_{0}}{K}-\frac{1}{2} \sigma^{2} t-\frac{1}{2} \rho_{t}^{2}\right)^{2}}{2 \sigma^{2} t+\rho_{t}^{2}}\right\} .
$$

The case $f(t)=t$ corresponds to the model with the uniform grid and $A_{1}=A_{1}(f)$.

We formulated Theorems 1.1 and 1.3 for convenience of references. The main result of this note is more general. It covers not only models with nonuniform grids but also models with pay-off functions satisfying the following

Assumption 3: $G: \mathbf{R}_{+} \rightarrow \mathbf{R}$ is a convex function such that $\left.G\right|_{I_{j}} \in C^{2}\left(I_{j}\right)$, where the intervals $I_{j}:=\left[K_{j-1}, K_{j}\right], j=1, \ldots, N, I_{N+1}:=\left[K_{N}, \infty[\right.$ with $0=K_{0}<K_{1}<\ldots<K_{N}<\infty$, and $G^{\prime \prime}(x) \leq \kappa\left(1+x^{m}\right)$ for some constants $m, M>0$. 
The pricing function

$$
\widehat{C}(t, x)=E G\left(x e^{\xi \rho_{t}-\frac{1}{2} \rho_{t}^{2}}\right), \quad t \in[0,1], x>0,
$$

solves the Cauchy problem

$$
\widehat{C}_{t}(t, x)+\frac{1}{2} \widehat{\sigma}_{t}^{2} x^{2} \widehat{C}_{x x}(t, x)=0, \quad \widehat{C}(1, x)=G(x) .
$$

Theorem 1.3 Suppose that for the scale function one of the Assumptions 1 or 2 is fulfilled and the pay-off function satisfies Assumption 3. Then

$$
E\left(V_{1}^{n}-V_{1}\right)^{2}=A_{1}(f) n^{-1}+o\left(n^{-1}\right), \quad n \rightarrow \infty,
$$

where $A_{1}(f)$ is given by the formula (1.12) with $\Lambda_{t}=E S_{t}^{4} \widehat{C}_{x x}^{2}\left(t, S_{t}\right)$.

The above result makes plausible the conjecture that the normalized difference $n^{1 / 2}\left(V_{1}^{n}-V_{1}\right)$ converges in law. Indeed, this is the case, see [4].

2. A point on the Grannan-Swindle paper. The Leland method based on the Black-Scholes formula is amongst a few practical recipes how to price options under transaction costs. It has an advantage to rely upon wellknown and well-understood formulae from the theory of frictionless markets. The method gave rise to a variety of other schemes. Of course, the precision of the resulting approximate hedging is an important issue, see [5], [2], [9], [11] and a survey [12] for related development.

The idea to parameterize the non-uniform grids by increasing functions and consider the family of strategies with the enlarged volatilities given by (1.10) is due to Grannan and Swindle, [3]. The mentioned paper claims that the asymptotics (1.16) holds for a general option with the pay-off of the form $G\left(S_{1}\right)$. In such a case the function $\widehat{C}(t, x)$ is the solution of the Cauchy problem

$$
\widehat{C}_{t}(t, x)+\frac{1}{2} \widehat{\sigma}_{t}^{2} x^{2} \widehat{C}_{x x}(t, x)=0, \quad \widehat{C}(1, x)=G(x) .
$$

To our opinion, the formulations and arguments given in [3] are not satisfactory. In particular, the hypothesis that for any nonnegative integers $m, n, p$

$$
\|\widehat{C}\|_{m, n, p}=\sup _{x>0, t \in[0,1]}\left[x^{m} \frac{\partial^{n+p} \widehat{C}(t, x)}{\partial x^{n} \partial t^{p}}\right]<\infty
$$

is not fulfilled for the call-option with $G(x)=(x-K)^{+}$(even for the uniform grid): explicit formulae show that derivatives of $\widehat{C}(t, x)$ have singularities at the point $(1, K)$. So, the mathematical results of the original paper [3] do not cover practically interesting cases. Nevertheless, the formula for $A_{1}(f)$ is used in numerical analysis of the approximate hedging error of call-options. Note also that the authors of [3] do not care about the eventual divergence of the integral (1.12) due to singularities of $1 / f^{\prime}$ which are not excluded by their assumptions. Neglecting the singularities may lead to an erroneous answer (recall the unfortunate error in Leland's paper corrected in [5] and which numerical aspects were discussed in [13], [14], [7]). That is why we 
are looking here for a rigorous proof to built a platform for further studies. The asymptotic analysis happens to be more involved comparatively with the arguments in [3]. Note that our assumption includes the case of the classical call-option.

The paper [3] contains another interesting idea: to minimize the functional $A_{1}(f)$ with respect to the scale $f$ in a hope to improve the performance of the strategy by an appropriate choice of the revision dates ${ }^{1}$. We alert the reader that the reduction to a classical variational problem is not correct as well as the derived Euler-Lagrange equation. That is why the whole paper [3] can be considered only as one giving useful heuristics but leaving open mathematical problems of practical importance.

3. Structure of the proof. The proof of Theorem 1.3 requires some preliminary work. In Section 2 we consider the process $V_{s}^{n}-\widehat{C}\left(s, S_{s}\right)$ which can be interpreted as a running deviation of the approximating portfolio process from the "theoretical" option price in the presence of transaction costs and which terminal value is the hedging error. We extract from this process a principal part which is the sum of two martingales $M^{1 n}$ and $M^{2 n}$ of a particular simple structure and a residual part split for convenience into sum of two processes $R^{1 n}$ and $R^{2 n}$. To prove the theorem it is sufficient to show that $\sqrt{n}\left\|M_{1}^{1 n}+M_{1}^{2 n}-A_{1}(f)\right\|_{L^{2}(\Omega)} \rightarrow 0$ (Proposition 2.2) and $\sqrt{n}\left\|R_{1}^{j n}\right\|_{L^{2}(\Omega)} \rightarrow 0$ as $n \rightarrow \infty$. However, having in mind applications to limit theorems for the residual we announce in Propositions 2.3 and 2.4 stronger results, namely, that $\sqrt{n}\left\|\sup _{s} R_{s}^{j n}\right\|_{L^{2}(\Omega)} \rightarrow 0$. Proofs of these three propositions are given in Sections $5-7$. Section 3 is devoted to derivation of estimates of partial derivatives of $\widehat{C}(t, x)$. Some auxiliary results are recalled in Section 4. The concluding Section 8 contains estimates of some Gaussian functionals.

We use the French-style terminology: "positive" - "strictly positive"; $\kappa$ stands for a constant which value is of no importance.

\section{Preparatory Manipulations}

First of all, we represent the deviation of the approximating portfolio from the pay-off in an integral form which is instructive how to proceed further.

In the sequel we need to define a number of stochastic processes. Since the terminal date plays a particular role (we do not include the final transaction), they will be defined on the interval $[0,1$ [ with an extension by continuity to its right extremity. With such a convention the identity in the following lemma holds also for $s=1$.

Lemma 2.1 We have the representation $V_{s}^{n}-\widehat{V}_{s}=F_{s}^{1 n}+F_{s}^{2 n}, s \in[0,1[$, where $\widehat{V}_{s}=\widehat{C}\left(s, S_{s}\right)$,

$$
F_{s}^{1 n}:=\sigma \int_{0}^{s} S_{t} \sum_{i=1}^{n}\left(\widehat{C}_{x}\left(t_{i-1}, S_{t_{i-1}}\right)-\widehat{C}_{x}\left(t, S_{t}\right)\right) I_{\left[t_{i-1}, t_{i}[\right.}(t) d W_{t},
$$

\footnotetext{
1 Even in the frictionless case the choice of an optimal scale to minimize the hedging error is an important and nontrivial problem, especially, for irregular payoff functions, see, e.g., [1] and references wherein.
} 


$$
F_{s}^{2 n}:=k_{0} \sqrt{\frac{2}{\pi}} \sigma \int_{0}^{s} S_{t}^{2} \widehat{C}_{x x}\left(t, S_{t}\right) \sqrt{f^{\prime}(t)} d t-\frac{k_{0}}{\sqrt{n}} \sum_{t_{i} \leq s}\left|\Delta \widehat{C}_{x}\left(t_{i}\right)\right| S_{t_{i}},
$$

with the abbreviation $\Delta \widehat{C}_{x}\left(t_{i}\right):=\widehat{C}_{x}\left(t_{i}, S_{t_{i}}\right)-\widehat{C}_{x}\left(t_{i-1}, S_{t_{i-1}}\right)$.

Proof. Using the expression (1.5) and applying the Ito formula to the increment $\widehat{C}\left(0, S_{0}\right)-\widehat{C}\left(s, S_{s}\right)$ we get that the difference $V_{s}^{n}-\widehat{V}_{s}$ is equal to

$$
F_{s}^{1 n}-\int_{0}^{s}\left(\widehat{C}_{t}\left(t, S_{t}\right)+\frac{1}{2} \sigma^{2} S_{t}^{2} \widehat{C}_{x x}\left(t, S_{t}\right)\right) d t-\frac{k_{0}}{\sqrt{n}} \sum_{t_{i} \leq s}\left|\Delta \widehat{C}_{x}\left(t_{i}\right) \cdot\right| S_{t_{i}}
$$

Since $\widehat{C}(t, x)$ solves the Cauchy problem (1.15), the integrand above is equal to $(1 / 2)\left(\sigma^{2}-\widehat{\sigma}_{t}^{2}\right) S_{t}^{2} \widehat{C}_{x x}\left(t, S_{t}\right)$. We conclude by substituting the expression (1.10) for $\widehat{\sigma}_{t}^{2}$.

Put (for $s \in[0,1[$ )

$$
\begin{aligned}
M_{s}^{1 n} & :=\frac{1}{2} \sigma^{2} \sum_{t_{i} \leq s} \widehat{C}_{x x}\left(t_{i-1}, S_{t_{i-1}}\right) S_{t_{i-1}}^{2}\left[\Delta t_{i}-\left(\Delta W_{t_{i}}\right)^{2}\right] \\
M_{s}^{2 n} & :=\sigma \frac{k_{0}}{\sqrt{n}} \sum_{t_{i} \leq s} \widehat{C}_{x x}\left(t_{i-1}, S_{t_{i-1}}\right) S_{t_{i-1}}^{2}\left[\sqrt{2 / \pi} \sqrt{\Delta t_{i}}-\left|\Delta W_{t_{i}}\right|\right]
\end{aligned}
$$

where $\Delta t_{i}:=t_{i}-t_{i-1}$ and $\Delta W_{t_{i}}:=W_{t_{i}}-W_{t_{i-1}}$.

We introduce also two residual processes $R_{s}^{j n}:=F_{s}^{j n}-M_{s}^{j n}, j=1,2$.

Since $\widehat{V}_{1}=G\left(S_{1}\right)$, Theorem 1.3 follows from the following two assertions:

Proposition $2.2 n E\left(M_{1}^{1 n}+M_{1}^{2 n}-A_{1}(f)\right)^{2} \rightarrow 0$ as $n \rightarrow \infty$.

Proposition 2.3 $n E \sup _{s \leq 1}\left(R_{s}^{1 n}\right)^{2} \rightarrow 0$ as $n \rightarrow \infty$.

Proposition $2.4 n E \sup _{s \leq 1}\left(R_{s}^{2 n}\right)^{2} \rightarrow 0$ as $n \rightarrow \infty$.

Remark. In fact, to prove the theorem, it would be sufficient to show that $n E\left(R_{1}^{j n}\right)^{2} \rightarrow 0$. However, the stronger property claimed above happens to be useful in a study of more delicate results on the asymptotic behavior of the hedging error.

For a process $X=\left(X_{t}\right)$ we denote by $X^{*}$ its maximal process. That is $X_{t}^{*}=\sup _{u \leq t}\left|X_{u}\right|$. In this (standard) notation the claims of Propositions 2.3 and 2.4 can be written as $n^{1 / 2}\left\|R_{1}^{j n *}\right\|_{L^{2}(\Omega)} \rightarrow 0, j=1,2$.

Note that the sum in the expression for $F_{1}^{n 2}=F_{1-}^{n 2}$ does not include the term with $i=n$. Having in mind singularities of derivatives at the maturity, it is convenient to isolate the last summands also in other sums and treat them separately.

Now we analyze the expressions for $F_{s}^{1 n}$ and $F_{s}^{1 n}$ by applying the Taylor expansion of the first order to the differences $\widehat{C}_{x}\left(t_{i-1}, S_{t_{i-1}}\right)-\widehat{C}_{x}\left(t, S_{t}\right)$ and $\widehat{C}_{x}\left(t_{i}, S_{t_{i}}\right)-\widehat{C}_{x}\left(t_{i-1}, S_{t_{i-1}}\right)$ at the point $\left(t_{i-1}, S_{t_{i-1}}\right)$. A short inspection of the resulting formulae using the helpful heuristics $\Delta S_{t} \approx \sigma S_{t} \Delta W_{t} \approx \sigma S_{t} \sqrt{d t}$ 
reveals that the main contributions in the first order Taylor approximations of increments originate from the derivatives of $\widehat{C}_{x}(t, x)$ in $x$. That is the principal terms of asymptotics are

$$
\begin{aligned}
P_{s}^{1 n} & :=\sigma \int_{0}^{s} \sum_{i=1}^{n-1} \widehat{C}_{x x}\left(t_{i-1}, S_{t_{i-1}}\right) S_{t_{i-1}}^{2}\left(1-S_{t} / S_{t_{i-1}}\right) S_{t} / S_{t_{i-1}} I_{\left[t_{i-1}, t_{i}[\right.}(t) d W_{t}, \\
P_{s}^{2 n} & :=k_{0} \sum_{t_{i} \leq s} \widehat{C}_{x x}\left(t_{i-1}, S_{t_{i-1}}\right) S_{t_{i-1}}^{2}\left[\sigma \sqrt{2 / \pi} \sqrt{f^{\prime}\left(t_{i-1}\right)} \Delta t_{i}-\left|S_{t_{i}} / S_{t_{i-1}}-1\right| / \sqrt{n}\right],
\end{aligned}
$$

where $s \in[0,1[$.

We write the first residual term $R_{s}^{1 n}=\left(P_{s}^{1 n}-M_{s}^{1 n}\right)+\left(F_{s}^{1 n}-P_{s}^{1 n}\right)$ in the following form:

$$
R_{s}^{1 n}:=\left(R_{s}^{1 M n}+R_{s}^{1 n n}-R_{s}^{1 t n}-(1 / 2) \tilde{R}_{s}^{1 n}\right) \sigma
$$

where

$$
\begin{aligned}
R_{s}^{1 M n} & :=\left(P_{s}^{1 n}-M_{s}^{1 n}\right) / \sigma \\
R_{s}^{1 n n} & :=I_{\left[t_{n-1}, t_{n}\right]}(s) \int_{t_{n-1}}^{s}\left(\widehat{C}_{x}\left(t_{n-1}, S_{t_{n-1}}\right)-\widehat{C}_{x}\left(t, S_{t}\right)\right) S_{t} d W_{t}, \\
R_{s}^{1 t n} & :=\int_{0}^{s} \sum_{i=1}^{n-1} \widehat{C}_{x t}\left(t_{i-1}, S_{t_{i-1}}\right)\left(t-t_{i-1}\right) S_{t} I_{\left[t_{i-1}, t_{i}[\right.}(t) d W_{t}, \\
\tilde{R}_{s}^{1 n} & :=\int_{0}^{s} \sum_{i=1}^{n-1} \tilde{U}_{t}^{i} I_{\left[t_{i-1}, t_{i}[\right.}(t) d W_{t}
\end{aligned}
$$

with

$$
\begin{aligned}
\tilde{U}_{t}^{i}= & \widehat{C}_{x x x}\left(\tilde{t}_{i-1}, \tilde{S}_{t_{i-1}}\right)\left(S_{t}-S_{t_{i-1}}\right)^{2} S_{t}+\widehat{C}_{x t t}\left(\tilde{t}_{i-1}, \tilde{S}_{t_{i-1}}\right)\left(t-t_{i-1}\right)^{2} S_{t} \\
& +2 \widehat{C}_{x x t}\left(\tilde{t}_{i-1}, \tilde{S}_{t_{i-1}}\right)\left(t-t_{i-1}\right)\left(S_{t}-S_{t_{i-1}}\right) S_{t} .
\end{aligned}
$$

The intermediate point $\left(\tilde{t}_{i-1}, \tilde{S}_{t_{i-1}}\right)$ in the interval connecting $\left(t_{i-1}, S_{t_{i-1}}\right)$ and $\left(t_{i}, S_{t_{i}}\right)$ can be chosen in such a way that the mapping $\omega \mapsto\left(\tilde{t}_{i-1}, \tilde{S}_{t_{i-1}}\right)$ is an $\mathcal{F}_{t_{i}}$-measurable random variable (for example, one cane take the first point on this interval for which the Taylor formula holds).

Notice that $\tilde{t}_{i-1} \in\left[t_{i-1}, t_{i}\right]$ and $\tilde{S}_{t_{i-1}} \in\left[S_{t_{i-1}}, S_{t}\right]$.

The structure of the above representation of $R^{1 n}$ is clear: the term $R^{1 n n}$ corresponds to the $n$-th revision interval (it will be treated separately because of singularities at the left extremity of the time interval), the term $R^{1 t n}$ involving the first derivatives of $\widehat{C}_{x}$ in $t$ at points $\left(t_{i-1}, S_{t_{i-1}}\right)$ comes from the Taylor formula, and the "tilde" term is due to the remainder of latter.

It is important to note that the integrals involving in the definition of $P^{1 n}$ depend only on the increments of the Wiener process on the intervals $\left[t_{i-1}, t_{i}\right]$ and, therefore, are independent on the $\sigma$-algebras $\mathcal{F}_{t_{i-1}}$. This helps to calculate the expectation of the squared sum: according to Lemma 4.1 below it is the sum of expectations of the squared terms. We define $P^{2 n}$ in a way to enjoy the same property. The second residual term includes 
the term $R^{2 n n}$ corresponding to the last revision interval; the term $R^{21 n}$ represents the approximation error arising from replacement of the integral by the Riemann sum; the remaining part of the residual we split in a natural way into summands $R_{22}^{n}$ and $R_{23}^{n}$. After these explanations we write the second residual term as follows:

We "telescope" the residual term $R_{s}^{2 n}=\left(P_{s}^{2 n}-M_{s}^{2 n}\right)+\left(F_{s}^{2 n}-P_{s}^{2 n}\right)$ in the following way:

$$
R_{s}^{2 n}=\left(R_{s}^{2 M n}+\sigma \sqrt{2 / \pi} R_{s}^{2 n n}+\sigma \sqrt{2 / \pi} R_{s}^{21 n}+R_{s}^{22 n}+R_{s}^{23 n}+R_{s}^{24 n}\right) k_{0},
$$

with

$$
\begin{aligned}
R_{s}^{2 M n} & =\left(P_{s}^{2 n}-M_{s}^{2 n}\right) / k_{0}, \\
R_{s}^{2 n n} & =I_{\left[t_{n-1}, t_{n}\right]}(s) \int_{t_{n-1}}^{s} S_{t}^{2} \widehat{C}_{x x}\left(t, S_{t}\right) \sqrt{f^{\prime}(t)} d t \\
R_{s}^{21 n} & =\sum_{i=1}^{n-1} \int_{t_{i-1}}^{t_{i}}\left(S_{t}^{2} \widehat{C}_{x x}\left(t, S_{t}\right) \sqrt{f^{\prime}(t)}-S_{t_{i-1}}^{2} \widehat{C}_{x x}\left(t_{i-1}, S_{t_{i-1}}\right) \sqrt{f^{\prime}\left(t_{i-1}\right)}\right) d t \\
R_{s}^{22 n} & =\frac{1}{\sqrt{n}} \sum_{t_{i} \leq s} \widehat{C}_{x x}\left(t_{i-1}, S_{t_{i-1}}\right)\left|S_{t_{i-1}}-S_{t_{i}}\right|\left(S_{t_{i-1}}-S_{t_{i}}\right), \\
R_{s}^{23 n} & =\frac{1}{\sqrt{n}} \sum_{t_{i} \leq s}[\ldots]_{i}\left(S_{t_{i}}-S_{t_{i-1}}\right) \\
R_{s}^{24 n} & =\frac{1}{\sqrt{n}} \sum_{t_{i} \leq s}[\ldots]_{i} S_{t_{i-1}},
\end{aligned}
$$

where

$$
[\ldots]_{i}=\widehat{C}_{x x}\left(t_{i-1}, S_{t_{i-1}}\right)\left|S_{t_{i}}-S_{t_{i-1}}\right|-\left|\widehat{C}_{x}\left(t_{i}, S_{t_{i}}\right)-\widehat{C}_{x}\left(t_{i-1}, S_{t_{i-1}}\right)\right| .
$$

\section{Convenient Representations, Explicit Formulae and Useful}

\section{Bounds}

\subsection{Representations of Derivatives in $x$}

We consider the function $\widehat{C}(t, x)$ defined by the formula (1.14), i.e.

$$
\widehat{C}(t, x)=\int_{-\infty}^{\infty} G\left(x e^{\rho_{t} y-\frac{1}{2} \rho_{t}^{2}}\right) \varphi(y) d y .
$$

To ensure that the integral is finite we suppose that $G: \mathbf{R}^{+} \rightarrow \mathbf{R}$ is of polynomial growth. We assume also that $G$ is a convex function. Automatically, $G$, being locally Liptsitz, admits a positive Radon-Nikodym derivative $G^{\prime}$. One can choose as $G^{\prime}$ the right derivative of $G$ which is increasing and has only a countable set of discontinuities. 
Our aim is to get appropriate estimates of partial derivatives of $\widehat{C}(t, x)$. To this end we introduce the function

$$
\bar{C}(x ; \rho)=\int_{-\infty}^{\infty} G\left(x e^{\rho y-\frac{1}{2} \rho^{2}}\right) \varphi(y) d y .
$$

Lemma 3.1 Suppose that $G$ is a convex function which Radon-Nikodym derivative $G^{\prime}$ has a polynomial growth. Then for $n=1,2,3,4$ we have the following representation:

$$
\frac{\partial^{n}}{\partial x^{n}} \bar{C}(x ; \rho)=\frac{1}{\rho^{n-1} x^{n-1}} \int_{-\infty}^{\infty} G^{\prime}\left(x e^{\rho y+\frac{1}{2} \rho^{2}}\right) P_{n-1}(y) \varphi(y) d y
$$

where

$$
\begin{aligned}
& P_{0}(y):=1 \\
& P_{1}(y):=y \\
& P_{2}(y):=y^{2}-\rho y-1 \\
& P_{3}(y):=y^{3}-3 \rho y^{2}+\left(2 \rho^{2}-3\right) y+3 \rho .
\end{aligned}
$$

Proof. Let us introduce the function

$$
\bar{G}(u ; \rho):=G\left(e^{-\rho u-\frac{1}{2} \rho^{2}}\right), \quad u \in \mathbf{R}, \rho>0 .
$$

Recall that the convolution of $\bar{G}$ and $\varphi$ is defined by the formula

$$
\bar{G} * \varphi(z ; \rho):=\int_{-\infty}^{\infty} \bar{G}(z-y ; \rho) \varphi(y) d y .
$$

The representation $\bar{C}(x ; \rho)=\bar{G} * \varphi\left(-\rho^{-1} \ln x ; \rho\right)$ allows us to calculate easily the derivatives in $x$.

Differentiating the convolution we get that

$$
\frac{\partial^{n}}{\partial z^{n}} \bar{G} * \varphi(z ; \rho)=\bar{G} * \varphi^{(n)}(z ; \rho)=\bar{G}^{\prime} * \varphi^{(n-1)}(z ; \rho) .
$$

Recalling that $\varphi^{(n)}(y)=(-1)^{n} H_{n}(y) \varphi(y)$ where $H_{n}$ is the Hermite polynomial of order $n$ we obtain the representations

$\frac{\partial^{n}}{\partial z^{n}} \bar{G} * \varphi(z ; \rho)=(-1)^{n} \rho \int_{-\infty}^{\infty} G^{\prime}\left(e^{-\rho(z-y)-\frac{1}{2} \rho^{2}}\right) e^{-\rho(z-y)-\frac{1}{2} \rho^{2}} H_{n-1}(y) \varphi(y) d y$

and

$\frac{\partial^{n}}{\partial z^{n}} \bar{G} * \varphi\left(-\rho^{-1} \ln x ; \rho\right)=(-1)^{n} \rho x \int_{-\infty}^{\infty} G^{\prime}\left(x e^{\rho y-\frac{1}{2} \rho^{2}}\right) e^{\rho y-\frac{1}{2} \rho^{2}} H_{n-1}(y) \varphi(y) d y$.

Changing the variable, we rewrite the last formula as

$$
\frac{\partial^{n}}{\partial z^{n}} \bar{G} * \varphi\left(-\rho^{-1} \ln x ; \rho\right)=(-1)^{n} \rho x \int_{-\infty}^{\infty} G^{\prime}\left(x e^{\rho y+\frac{1}{2} \rho^{2}}\right) H_{n-1}(y+\rho) \varphi(y) d y
$$


The first four derivatives of the function $f(g()$.$) at the point x$ are given by the formulae

$$
\begin{aligned}
\frac{d}{d x} f(g(x))= & f_{g}^{\prime}(x) g^{\prime}(x), \\
\frac{d^{2}}{d x^{2}} f(g(x))= & f_{g}^{\prime \prime}(x)\left(g^{\prime}(x)\right)^{2}+f_{g}^{\prime}(x) g^{\prime \prime}(x), \\
\frac{d^{3}}{d x^{3}} f(g(x))= & f_{g}^{(3)}(x)\left(g^{\prime}(x)\right)^{3}+3 f_{g}^{\prime \prime}(x) g^{\prime \prime}(x) g^{\prime}(x)+f_{g}^{\prime}(x) g^{(3)}(x), \\
\frac{d^{4}}{d x^{4}} f(g(x))= & f_{g}^{(4)}(x)\left(g^{\prime}(x)\right)^{4}+6 f_{g}^{(3)}(x) g^{\prime \prime}(x)\left(g^{\prime}(x)\right)^{2}+3 f_{g}^{\prime \prime}(x)\left(g^{\prime \prime}(x)\right)^{2} \\
& +4 f_{g}^{\prime \prime}(x) g^{(3)}(x) g^{\prime}(x)+f_{g}^{\prime}(x) g^{(4)}(x),
\end{aligned}
$$

where we use the abbreviations $f_{g}^{\prime}(x):=f^{\prime}(g(x)), f_{g}^{\prime \prime}(x):=f^{\prime \prime}(g(x))$ etc.

For $g(x)=-\rho^{-1} \ln x$ the $m$-th derivative $g^{(m)}(x)=(-1)^{m}(m-1) ! \rho^{-1} x^{-m}$. Applying the above formulae with $f=\bar{G} * \varphi$ and $f_{g}^{(n)}(x)$ given by the righthand side of (3.4) we obtain the assertion of the lemma with

$$
\begin{aligned}
& P_{0}(y)=H_{0}(y+\rho), \\
& P_{1}(y)=H_{1}(y+\rho)-\rho H_{0}(y+\rho), \\
& P_{2}(y)=H_{2}(y+\rho)-3 \rho H_{1}(y+\rho)+2 \rho^{2} H_{0}(y+\rho), \\
& P_{3}(y)=H_{3}(y+\rho)-6 \rho H_{2}(y+\rho)+11 \rho^{2} H_{1}(y+\rho)+6 \rho^{3} H_{0}(y+\rho) .
\end{aligned}
$$

Since $H_{0}(y)=1, H_{1}(y)=y, H_{2}(y)=y^{2}-1, H_{3}(y)=y^{3}-3 y$, these formulae can be re-written as in the statement of the lemma.

Remark. Using the well-known combinatorial formula for the $n$-th derivative of $f(g(x))$ (see, e.g., Th. III.21 in the textbook [10]) one can check easily that the representation (3.3) holds for each $n$ with a certain polynomial $P_{n-1}$ of two variables, $y$ and $\rho$, of order $n-1$ and the coefficient at $y^{n-1}$ equal to unit.

It follows from the above lemma and accompanying remark that in the case where $G^{\prime}(x) \leq \kappa\left(1+x^{p}\right)$

$$
\frac{\partial^{n}}{\partial x^{n}} \widehat{C}(t, x) \leq \kappa_{n} \frac{\left(1+x^{p}\right)}{x^{n-1}(1-t)^{(n-1) / 2}} .
$$

In particular, if $G^{\prime}$ is bounded we have that

$$
\frac{\partial^{n}}{\partial x^{n}} \widehat{C}(t, x) \leq \kappa_{n} \frac{1}{x^{n-1}(1-t)^{(n-1) / 2}} .
$$

Lemma 3.2 Suppose that $G$ is a convex function which Radon-Nikodym derivative $G^{\prime}$ has a polynomial growth. Then $\widehat{C}_{x}\left(t, S_{t}\right) \rightarrow G^{\prime}\left(S_{1}\right)$ as $t \rightarrow 1$ almost surely and in any $L^{p}(\Omega), p<\infty$.

Proof. From the representation (3.3) with $n=1$ it follows that

$$
\widehat{C}_{x}\left(t, S_{t}\right)=\int_{-\infty}^{\infty} G^{\prime}\left(S_{t} e^{\rho_{t} y+\frac{1}{2} \rho_{t}^{2}}\right) \varphi(y) d y .
$$


Since the distribution of $S_{1}$ is continuous, the set $\Omega_{0}$ of $\omega$ for which $S_{1}(\omega)$ belongs to the (countable) set of discontinuities of $G^{\prime}$ has zero probability. Outside $\Omega_{0}$ we apply to the integral the Lebesgue theorem on dominated convergence using the assumption that $G^{\prime}$ has a polynomial growth. To get the convergence in $L^{p}(\Omega)$ we also apply the Lebesgue theorem but now to the expectation. Its condition holds because $S_{1}^{*}$ is integrable in any power.

\subsection{Representations of Mixed Derivatives}

Explicit formulae for derivatives involving the variable $t$ are more cumbersome but also easy to obtain.

Let us define the operator $T$ transforming the polynomial $P(y ; \rho)$ into the polynomial

$$
T P(y ; \rho)=\left(y P(y ; \rho)-P_{y}(y ; \rho)\right)(y+\rho)+\rho P_{\rho}(y ; \rho)-P(y ; \rho) .
$$

Lemma 3.3 Suppose that $G$ is an increasing convex function which RadonNikodym derivative $G^{\prime}$ has a polynomial growth. Then we have the following formulae:

$$
\begin{aligned}
\widehat{C}_{t}(t, x)= & -\frac{\widehat{\sigma}_{t}^{2} x}{2 \rho_{t}} \int_{-\infty}^{\infty} G^{\prime}\left(x e^{\rho_{t} y+\frac{1}{2} \rho_{t}^{2}}\right) P_{1}(y) \varphi(y) d y \\
\widehat{C}_{x t}(t, x)= & -\frac{\widehat{\sigma}_{t}^{2}}{2 \rho_{t}^{2}} \int_{-\infty}^{\infty} G^{\prime}\left(x e^{\rho_{t} y+\frac{1}{2} \rho_{t}^{2}}\right) T P_{0}\left(y ; \rho_{t}\right) \varphi(y) d y \\
\widehat{C}_{x x t}(t, x)= & -\frac{\widehat{\sigma}_{t}^{2}}{2 \rho_{t}^{3} x} \int_{-\infty}^{\infty} G^{\prime}\left(x e^{\rho_{t} y+\frac{1}{2} \rho_{t}^{3} x}\right)\left(T P_{1}\left(y ; \rho_{t}\right)+P_{1}(y)\right) \varphi(y) d y \\
\widehat{C}_{t t}(t, x)= & -\left(\frac{\left(\widehat{\sigma}_{t}^{2}\right)^{\prime}}{2 \rho_{t}}+\frac{\widehat{\sigma}_{t}^{4}}{4 \rho_{t}^{3}}\right) x \int_{-\infty}^{\infty} G^{\prime}\left(x e^{\rho_{t} y+\frac{1}{2} \rho_{t}^{2}}\right) P_{1}(y) \varphi(y) d y \\
& +\frac{\widehat{\sigma}_{t}^{4} x}{4 \rho_{t}^{3}} \int_{-\infty}^{\infty} G^{\prime}\left(x e^{\rho_{t} y+\frac{1}{2} \rho_{t}^{2}}\right) T P_{1}\left(y ; \rho_{t}\right)(y) \varphi(y) d y, \\
\widehat{C}_{x t t}(t, x)= & -\left(\frac{\left(\widehat{\sigma}_{t}^{2}\right)^{\prime}}{2 \rho_{t}^{2}}+\frac{\widehat{\sigma}_{t}^{4}}{2 \rho_{t}^{4}}\right) \int_{-\infty}^{\infty} G^{\prime}\left(x e^{\rho_{t} y+\frac{1}{2} \rho_{t}^{2}}\right) T P_{0}\left(y ; \rho_{t}\right) \varphi(y) d y \\
& +\frac{\widehat{\sigma}_{t}^{4}}{2 \rho_{t}^{4}} \int_{-\infty}^{\infty} G^{\prime}\left(x e^{\rho_{t} y+\frac{1}{2} \rho_{t}^{2}}\right) T^{2} P_{0}\left(y ; \rho_{t}\right) \varphi(y) d y
\end{aligned}
$$

where $P_{j}(y)$ are polynomials defined in Lemma 3.3. In accordance to the definition of the operator $T$,

$$
\begin{aligned}
& T P_{0}(y)=y^{2}+\rho y-1, \\
& T P_{1}(y)=y^{3}+\rho y^{2}-2 y-\rho .
\end{aligned}
$$

Proof. Differentiating under the sign of integral in (3.2) and making a linear change of variables we obtain the representation

$$
\bar{C}_{\rho}(x ; \rho)=x \int_{-\infty}^{\infty} G^{\prime}\left(x e^{\rho y+\frac{1}{2} \rho^{2}}\right) y \varphi(y) d y .
$$


Since $\rho_{t}^{\prime}=-\sigma_{t}^{2} /\left(2 \rho_{t}\right)$, the formula for $\widehat{C}_{t}(t, x)$ follows obviously.

Using the change of variable

$$
z(y ; x, \rho)=x e^{\rho y+\frac{1}{2} \rho^{2}}
$$

with the inverse

$$
y(z ; x, \rho)=\frac{1}{\rho} \ln \frac{z}{x}-\frac{1}{2} \rho
$$

and differentiating under the sign of integral we get that

$\frac{\partial}{\partial \rho} \int_{-\infty}^{\infty} G^{\prime}\left(x e^{\rho y+\frac{1}{2} \rho^{2}}\right) P(y ; \rho) \varphi(y) d y=\frac{1}{\rho} \int_{-\infty}^{\infty} G^{\prime}\left(x e^{\rho y+\frac{1}{2} \rho^{2}}\right) T P(y ; \rho) \varphi(y) d y$.

This identity help us to derive the formulae for $\widehat{C}_{x t}(t, x)$ and $\widehat{C}_{x x t}(t, x)$ from the representation (3.3) and also get the formulae for $\widehat{C}_{t t}(t, x)$ and $\widehat{C}_{x t t}(t, x)$ by differentiation of those for $\widehat{C}_{t}(t, x)$ and $\widehat{C}_{x t}(t, x)$.

From the above lemma we have the following bounds:

Lemma 3.4 Suppose that one of the Assumptions 1 or 2 is fulfilled and $G^{\prime}$ has a polynomial growth. Then

$$
\begin{aligned}
\left|\widehat{C}_{x t}(t, x)\right| & \leq \kappa \frac{1}{1-t}\left(1+x^{m}\right), \\
\left|\widehat{C}_{x x t}(t, x)\right| & \leq \kappa \frac{1}{(1-t)^{3 / 2}} \frac{1}{x}\left(1+x^{m}\right), \\
\left|\widehat{C}_{x t t}(t, x)\right| & \leq \kappa \frac{1}{(1-t)^{2}}\left(1+x^{m}\right) .
\end{aligned}
$$

Proof. Under the Assumption 1 both $\widehat{\sigma}_{t}^{2}$ and $\left|\left(\widehat{\sigma}_{t}^{2}\right)^{\prime}\right|=\kappa\left|f^{\prime \prime}(t)\right| / \sqrt{f^{\prime}(t)}$ are bounded and the statement is obvious. Under the Assumption 2, i.e. when $f(t)=1-(1-t)^{1 / \beta}, \beta>1$, direct calculations lead to the bounds

$$
\frac{\widehat{\sigma}_{t}^{2}}{\rho_{t}^{2}} \leq \kappa \frac{1}{1-t}, \quad \frac{\left|\left(\widehat{\sigma}_{t}^{2}\right)^{\prime}\right|}{\rho_{t}^{2}} \leq \kappa \frac{1}{(1-t)^{2}},
$$

implying required estimates.

\subsection{Sharper Estimates of Partial Derivatives}

For our analysis we need also more precise estimates requiring further hypotheses on $G$.

Put

$$
\Sigma_{N}(x, \rho):=\sum_{j=1}^{N} \exp \left\{-\frac{1}{2} \frac{\ln ^{2}\left(K_{j} / x\right)}{\rho^{2}}\right\}
$$

with the convention $\Sigma_{0}(x, \rho):=0$. 
Lemma 3.5 Under Assumption 3 there is a constant $\kappa$ such that for any $\rho \in[0, \sigma]$

$$
0 \leq \bar{C}_{x x}(x, \rho) \leq \kappa \frac{1}{\rho x^{3 / 2}} \Sigma_{N}(x, \rho)+\kappa\left(1+x^{m}\right)
$$

Proof. Put

$$
\delta_{j}:=\frac{1}{\rho} \ln \frac{K_{j}}{x}-\frac{1}{2} \rho .
$$

Integrating by parts on the closed intervals with the extremities $\delta_{j}$ we obtain that

$$
\begin{aligned}
\int_{0}^{\infty} G^{\prime}\left(x e^{\rho y+\frac{1}{2} \rho^{2}}\right) y \varphi(y) d y= & -\left.\sum_{j=0}^{N} G^{\prime}\left(x e^{\rho y+\frac{1}{2} \rho^{2}}\right) \varphi(y)\right|_{\delta_{j}+} ^{\delta_{j+1}-} \\
& +\rho x \int_{-\infty}^{\infty} G^{\prime \prime}\left(x e^{\rho y+\frac{1}{2} \rho^{2}}\right) e^{\rho y+\frac{1}{2} \rho^{2}} \varphi(y) d y
\end{aligned}
$$

Clearly,

$$
-\left.\sum_{j=0}^{N} G^{\prime}\left(x e^{\rho y+\frac{1}{2} \rho^{2}}\right) \varphi(y)\right|_{\delta_{j}+} ^{\delta_{j+1}-}=\sum_{j=1}^{N}\left(G^{\prime}\left(K_{j}+\right)-\left(G^{\prime}\left(K_{j}-\right)\right) \varphi\left(\delta_{j}\right) .\right.
$$

Due to assumed convexity of $G$ the summands in the right-hand side are positive and dominated by

$$
G^{\prime}\left(K_{N}+\right) \varphi\left(\delta_{j}\right)=G^{\prime}\left(K_{N}+\right) \frac{1}{\sqrt{2 \pi}} e^{-\rho^{2} / 8} \frac{K_{j}{ }^{1 / 2}}{x^{1 / 2}} \exp \left\{-\frac{1}{2} \frac{\ln ^{2}\left(K_{j} / x\right)}{\rho^{2}}\right\} .
$$

Due to the polynomial growth condition on $G^{\prime \prime}$ in the Assumption 3

$$
\int_{-\infty}^{\infty} G^{\prime \prime}\left(x e^{\rho y+\frac{1}{2} \rho^{2}}\right) e^{\rho y+\frac{1}{2} \rho^{2}} \varphi(y) d y \leq \kappa\left(1+x^{m}\right) .
$$

Combining the above estimates we infer that

$0 \leq \int_{0}^{\infty} G^{\prime}\left(x e^{\rho y+\frac{1}{2} \rho^{2}}\right) y \varphi(y) d y \leq \frac{\kappa}{x^{1 / 2}} \sum_{j=1}^{N} \exp \left\{-\frac{1}{2} \frac{\ln ^{2}\left(K_{j} / x\right)}{\rho^{2}}\right\}+\kappa \rho x\left(1+x^{m}\right)$

The claim follows now from the representation (3.3) for $n=2$.

Lemma 3.6 Under Assumption 3 there is a constant $\kappa$ such that for any $\rho \in] 0, \sigma]$

$$
\begin{array}{r}
\left|\bar{C}_{x x x}(x, \rho)\right| \leq \kappa \frac{1}{\rho^{2} x^{5 / 2}} \Sigma_{N}(x, \rho)+\kappa \frac{1}{\rho x}\left(1+x^{m}\right), \\
\left|\bar{C}_{x x x x}(x, \rho)\right| \leq \kappa \frac{1}{\rho^{3} x^{7 / 2}} \Sigma_{N}(x, \rho)+\kappa \frac{1}{\rho^{2} x^{2}}\left(1+x^{m}\right),
\end{array}
$$


Proof. For $Q_{n}(y)=H_{n}(y)$ the estimate

$$
\left|\int_{0}^{\infty} G^{\prime}\left(x e^{\rho y+\frac{1}{2} \rho^{2}}\right) Q_{n}(y) \varphi(y) d y\right| \leq \kappa \frac{1}{x^{1 / 2}} \Sigma_{N}(x, \rho)+\kappa \rho x\left(1+x^{m}\right)
$$

can be obtained by the same argument as above. The Hermite polynomials $H_{n}(y)$ form a basis in the linear space of polynomials in $y$. It follows that this estimate holds when $Q_{n}(y)=y^{n}$ and, hence, for any polynomial which coefficients are functions of $\rho$ bounded on $[0, \sigma]$. With this we conclude using the representation (3.3).

Using the estimate (3.13) we obtain from Lemma 3.3 the following:

Lemma 3.7 Under Assumption 3 on the pay-off function $G$ there is a constant $\kappa$ such that for any $t \in[0,1[$

$$
\begin{aligned}
\left|\widehat{C}_{t}(t, x)\right| & \leq \kappa \frac{\widehat{\sigma}_{t}^{2} x^{1 / 2}}{\rho_{t}} \Sigma_{N}\left(x, \rho_{t}\right)+\kappa \widehat{\sigma}_{t}^{2} x^{2}\left(1+x^{m}\right), \\
\left|\widehat{C}_{x t}(t, x)\right| & \leq \kappa \frac{\widehat{\sigma}_{t}^{2}}{\rho_{t}^{2} x^{1 / 2}} \Sigma_{N}\left(x, \rho_{t}\right)+\kappa \frac{\widehat{\sigma}_{t}^{2}}{\rho_{t}} x\left(1+x^{m}\right), \\
\left|\widehat{C}_{x x t}(t, x)\right| & \leq \kappa \frac{\widehat{\sigma}_{t}^{2}}{\rho_{t}^{3} x^{3 / 2}} \Sigma_{N}\left(x, \rho_{t}\right)+\kappa \frac{\widehat{\sigma}_{t}^{2}}{\rho_{t}^{2}}\left(1+x^{m}\right), \\
\left|\widehat{C}_{t t}(t, x)\right| & \leq \kappa\left(\frac{\left(\widehat{\sigma}_{t}^{2}\right)^{\prime}}{2 \rho_{t}}+\frac{\widehat{\sigma}_{t}^{4}}{4 \rho_{t}^{3}}\right)\left(x^{1 / 2} \Sigma_{N}\left(x, \rho_{t}\right)+\rho_{t} x^{2}\left(1+x^{m}\right)\right), \\
\left|\widehat{C}_{x t t}(t, x)\right| & \leq \kappa\left(\frac{\left(\widehat{\sigma}_{t}^{2}\right)^{\prime}}{2 \rho_{t}}+\frac{\widehat{\sigma}_{t}^{4}}{2 \rho_{t}^{4}}\right)\left(\frac{1}{x^{1 / 2}} \Sigma_{N}\left(x, \rho_{t}\right)+\rho_{t} x\left(1+x^{m}\right)\right) .
\end{aligned}
$$

\subsection{Call Option: Explicit Formulae}

For the classical call option with $G(x)=(x-K)^{+}$the derivatives we need can be given explicitly. In particular,

$$
\begin{aligned}
\widehat{C}_{x}(t, x) & =\Phi(\widehat{d}(t, x)), \\
\widehat{C}_{x x}(t, x) & =\frac{1}{x \rho_{t}} \varphi(\widehat{d}(t, x)),
\end{aligned}
$$

where

$$
\widehat{d}(t, x):=\frac{1}{\rho_{t}} \ln \frac{x}{K}+\frac{1}{2} \rho_{t} .
$$

To get the expression for the function $\Lambda_{t}=E S_{t}^{4} \widehat{C}_{x x}^{2}\left(t, S_{t}\right)$ from Theorem 1.11 we use the following easily verified formula.

Let $\xi \in \mathcal{N}(0,1)$ and let $a \neq 0, b, c$ be arbitrary constants. Then

$$
E e^{c \xi} e^{-(a \xi+b)^{2}}=\frac{1}{\sqrt{2 a^{2}+1}} \exp \left\{-\frac{\tilde{b}^{2}}{2 a^{2}+1}+\tilde{b}^{2}-b^{2}\right\} .
$$

where $\tilde{b}:=b-c /(2 a)$. 
The distribution of the random variable $2 \pi S_{t}^{p} \widehat{C}_{x x}^{2}\left(t, S_{t}\right)$ is the same as of

$$
S_{0}^{p-2} e^{-\frac{1}{2}(p-2) \sigma^{2} t} \rho_{t}^{-2} e^{c_{t} \xi} e^{-\left(a_{t} \xi+b_{t}\right)^{2}}
$$

where $c_{t}=(p-2) \sigma t^{1 / 2}, a_{t}=\frac{1}{\rho_{t}} \sigma t^{1 / 2}$,

$$
b_{t}=\frac{1}{\rho_{t}}\left(\ln \frac{S_{0}}{K}-\frac{1}{2} \sigma^{2} t\right)+\frac{1}{2} \rho_{t}, \quad \tilde{b}_{t}=b_{t}-\frac{1}{2}(p-2) \rho_{t} .
$$

Since

$$
\tilde{b}_{t}^{2}-b_{t}^{2}=-(p-2)\left[\left(\ln \frac{S_{0}}{K}-\frac{1}{2} \sigma^{2} t\right)+\rho_{t}^{2}-\frac{1}{4} p \rho_{t}^{2}\right],
$$

we obtain from above that

$$
E S_{t}^{p} \widehat{C}_{x x}^{2}\left(t, S_{t}\right)=\frac{1}{2 \pi \rho_{t}} \frac{K^{p-2}}{\sqrt{2 \sigma^{2} t+\rho_{t}^{2}}} e^{-B_{t}},
$$

where

$$
B_{t}:=\frac{\left(\ln \frac{S_{0}}{K}-\frac{1}{2} \sigma^{2} t-\frac{1}{2}(p-3) \rho_{t}^{2}\right)^{2}}{2 \sigma^{2} t+\rho_{t}^{2}}-\frac{(p-2)(p-4)}{4} \rho_{t}^{2} .
$$

In particular, with $p=4$, we have:

$$
\Lambda_{t}=\frac{1}{2 \pi \rho_{t}} \frac{K^{2}}{\sqrt{2 \sigma^{2} t+\rho_{t}^{2}}} \exp \left\{-\frac{\left(\ln \frac{S_{0}}{K}-\frac{1}{2} \sigma^{2} t-\frac{1}{2} \rho_{t}^{2}\right)^{2}}{2 \sigma^{2} t+\rho_{t}^{2}}\right\} .
$$

\subsection{Bounds for Expectations}

Using (3.15) we obtain from Lemma 3.5 - 3.7 and (3.10) the bounds which will be used in the proof of the main theorem.

Lemma 3.8 Suppose that one of the Assumptions 1 or 2 is fulfilled and $G$ satisfies the Assumption 3. Then

$$
\begin{aligned}
E S_{t}^{p} \widehat{C}_{x x}^{2 m}\left(t, S_{t}\right) & \leq \kappa \frac{1}{(1-t)^{m-1 / 2}}, \\
E S_{t}^{p} \widehat{C}_{x t}^{2 m}\left(t, S_{t}\right) & \leq \kappa \frac{1}{(1-t)^{2 m-1 / 2}}, \\
E S_{t}^{p} \widehat{C}_{x x x}^{2 m}\left(t, S_{t}\right) & \leq \kappa \frac{1}{(1-t)^{2 m-1 / 2}}, \\
E S_{t}^{p} \widehat{C}_{x x t}^{2 m}\left(t, S_{t}\right) & \leq \kappa \frac{1}{(1-t)^{3 m-1 / 2}}, \\
E S_{t}^{p} \widehat{C}_{x x x x}^{2 m}\left(t, S_{t}\right) & \leq \kappa \frac{1}{(1-t)^{3 m-1 / 2}},
\end{aligned}
$$

where the constant $\kappa$ depends on $p$ and $m$. In particular,

$$
\Lambda_{t} \leq \kappa \frac{1}{\sqrt{1-t}}
$$




\section{Tools}

In our computations we shall use frequently the following two assertions. The first one is a standard fact on square integrable martingales in discrete time.

Lemma 4.1 Let $M=\left(M_{i}\right)$ be a square-integrable martingale with respect to a filtration $\left(\mathcal{G}_{i}\right), i=0, \ldots, k$, and let $X=\left(X_{i}\right)$ be a predictable process with $E X^{2} \cdot\langle M\rangle_{k}<\infty$. Then

$$
E\left(X \cdot M_{k}\right)^{2}=E X^{2} \cdot\langle M\rangle_{k}=\sum_{i=1}^{k} E X_{i}^{2}\left(\Delta M_{i}\right)^{2},
$$

where, as usual, $\Delta\langle M\rangle_{i}:=E\left(\left(\Delta M_{i}\right)^{2} \mid \mathcal{G}_{i-1}\right)$,

$$
X \cdot M_{k}:=\sum_{i=1}^{k} X_{i} \Delta M_{i}, \quad X^{2} \cdot\langle M\rangle_{k}:=\sum_{i=1}^{k} X_{i}^{2}\langle M\rangle_{i} .
$$

Lemma 4.2 Suppose that $g^{\prime}, f^{\prime} \in C([0,1])$. Let $p>0$ and $a \geq 0$. Then

$$
\sum_{i=1}^{n-1} \frac{\left(\Delta t_{i}\right)^{p+a}}{\left(1-t_{i}\right)^{p}}= \begin{cases}O\left(n^{1-p-a}\right), & p<1 \\ O\left(n^{-a} \ln n\right), & p=1 \\ O\left(n^{-a}\right), & p>1\end{cases}
$$

If $g(t)=1-(1-t)^{\beta}, \beta \geq 1$, then

$$
\sum_{i=1}^{n-1} \frac{\left(\Delta t_{i}\right)^{p+a}}{\left(1-t_{i}\right)^{p}}= \begin{cases}O\left(n^{1-p-a}\right), & p<1+a(\beta-1) \\ O\left(n^{-a \beta} \ln n\right), & p=1+a(\beta-1) \\ O\left(n^{-a}\right), & p>1+a(\beta-1) .\end{cases}
$$

Proof. We consider first the case where $g^{\prime}, f^{\prime} \in C([0,1])$, i.e. $g^{\prime}$ is not only bounded but also bounded away from zero. By the finite increments formula $\Delta t_{i}=g^{\prime}\left(x_{i}\right) n^{-1}$ where $x_{i} \in[(i-1) / n, i / n]$ and, hence, $\Delta t_{i} \leq$ const $n^{-1}$. Applying again the finite increments formula and taking into account that $\min g^{\prime}(t)>0$, it is easy to check that there is a constant $c$ such that

$$
\frac{1-t_{i-1}}{1-t_{i}} \leq c, \quad 1 \leq i \leq n-1
$$

Thus,

$$
\sum_{i=1}^{n-1} \frac{\Delta t_{i}}{\left(1-t_{i}\right)^{p}} \leq c \sum_{i=1}^{n-1} \frac{\Delta t_{i}}{\left(1-t_{i-1}\right)^{p}} \leq c \int_{0}^{t_{n-1}} \frac{d t}{(1-t)^{p}}
$$

Since

$$
n^{-1} \min g^{\prime}(t) \leq 1-g(1-1 / n) \leq n^{-1} \max g^{\prime}(t),
$$

the asymptotic of the last integral is $O(1)$, if $p<1$ (the integral converges), $O(\ln n)$, if $p=1$, and $O\left(n^{p-1}\right)$, if $p>1$, This implies the claimed property. In the second case where $g(t)=1-(1-t)^{\beta}, \beta \geq 1$, we have

$$
\sum_{i=1}^{n-1} \frac{\left(\Delta t_{i}\right)^{p+a}}{\left(1-t_{i}\right)^{p}}=\frac{\beta^{p+a}}{n^{p-1+a}} \sum_{i=1}^{n-1} \frac{\left(1-x_{i}\right)^{(\beta-1)(p+a)}}{(1-i / n)^{\beta p}} \frac{1}{n} .
$$


The sum in the right-hand side is dominated, up to a multiplicative constant, by

$$
\sum_{i=1}^{n-1} \frac{1}{(1-(i-1) / n)^{p+a-\beta a}} \frac{1}{n} \leq \int_{0}^{1-1 / n} \frac{d t}{(1-t)^{p+a-\beta a}} .
$$

Using the explicit formulae for the integral we infer that the required property holds whatever are the parameters $p>0, a \geq 0$, and $\beta \geq 1$.

\section{Analysis of the Principal Terms: Proof of Proposition 2.2}

Since $E\left(M_{1}^{1 n}+M_{1}^{2 n}\right)=0$, we need to verify that $n E\left(M_{1}^{1 n}+M_{1}^{2 n}\right)^{2} \rightarrow A_{1}(f)$ as $n \rightarrow \infty$.

Recall that $E\left(\xi^{2}-1\right)^{2}=2$ and $E|\xi|^{3}=2 E|\xi|=2 \sqrt{2 / \pi}$ for $\xi \in \mathcal{N}(0,1)$. Using Lemma 4.1 we obtain the representation

$$
\begin{aligned}
n E\left(M_{1}^{1 n}+M_{1}^{2 n}\right)^{2}= & \frac{\sigma^{4}}{2} n \sum_{i=1}^{n-1} \Lambda_{t_{i-1}}\left(\Delta t_{i}\right)^{2}+k_{0} \sigma^{3} \sqrt{\frac{2}{\pi}} n^{1 / 2} \sum_{i=1}^{n-1} \Lambda_{t_{i-1}}\left(\Delta t_{i}\right)^{3 / 2} \\
& +k_{0}^{2} \sigma^{2}\left(1-\frac{2}{\pi}\right) \sum_{i=1}^{n-1} \Lambda_{t_{i-1}} \Delta t_{i} .
\end{aligned}
$$

By the finite increments formula $\Delta t_{i}=g(i / n)-g((i-1) / n)=g^{\prime}\left(x_{i}\right) / n$ where $x_{i} \in[(i-1) / n, i / n]$. We substitute this expression into the sums above. Let us introduce the function $F_{n}$ (depending on $p$ ) by the formula

$$
F_{n}(t):=\sum_{i=1}^{n-1} \Lambda_{g((i-1) / n)}\left[g^{\prime}\left(x_{i}\right)\right]^{p} I_{[(i-1) / n, i / n[}(t) .
$$

For $p \geq 1$ we have:

$$
\sum_{i=1}^{n-1} \Lambda_{g((i-1) / n)}\left[g^{\prime}\left(x_{i}\right)\right]^{p} \frac{1}{n}=\int_{0}^{1} F_{n}(t) d t \rightarrow \int_{0}^{1} \Lambda_{g(t)}\left[g^{\prime}(t)\right]^{p} d t .
$$

The needed uniform integrability of the sequence $\left\{F_{n}\right\}$ with respect to the Lebesgue measure follows from the de la Vallee-Poussin criterion because the estimate $\Lambda_{t} \leq \kappa(1-t)^{-1 / 2}$ and the boundedness of $g^{\prime}$ imply that

$$
\int_{0}^{1} F_{n}^{3 / 2}(t) d t \leq \mathrm{const} \int_{0}^{1} \frac{d g(t)}{(1-g(t))^{3 / 4}}=\mathrm{const} \int_{0}^{1} \frac{d s}{(1-s)^{3 / 4}}<\infty
$$

By the change of variable, taking into account that $g^{\prime}(t)=1 / f^{\prime}(g(t))$, we transform the limiting integral into the form used in the formulations of the theorem:

$$
\int_{0}^{1} \Lambda_{g(t)}\left[g^{\prime}(t)\right]^{p} d t=\int_{0}^{1} \Lambda_{g(t)}\left[g^{\prime}(t)\right]^{p-1} d g(t)=\int_{0}^{1} \Lambda_{t}\left[f^{\prime}(t)\right]^{1-p} d t .
$$

The claimed property on the convergence of $n^{1 / 2}\left(M_{1}^{1 n}+M_{1}^{2 n}\right)$ to $A_{1}(f)$ in $L^{2}$-norm is verified. 


\section{Analysis of the Residual $R^{1 n}$}

In this subsection we give a proof of Proposition 2.3.

1. To check the convergence of the sequence $n^{1 / 2} R_{1}^{1 M n *}$ to zero in $L^{2}$ it is convenient to introduce the "intermediate" process

$$
\bar{M}_{s}^{1 n}:=\sigma^{2} \int_{0}^{s} \sum_{i=1}^{n-1} \widehat{C}_{x x}\left(t_{i-1}, S_{t_{i-1}}\right) S_{t_{i-1}}^{2}\left(W_{t_{i-1}}-W_{t}\right) I_{\left[t_{i-1}, t_{i}[\right.}(t) d W_{t} .
$$

The difference $P^{1 n}-\bar{M}^{1 n}$ is a square integrable martingale and

$E\left(P_{1}^{1 n}-\bar{M}_{1}^{1 n}\right)^{2}=\sigma^{2} \sum_{i=1}^{n-1} \Lambda_{t_{i-1}} \int_{t_{i-1}}^{t_{i}} E\left[\left(\frac{S_{t}}{S_{t_{i-1}}}-1\right) \frac{S_{t}}{S_{t_{i-1}}}-\sigma\left(W_{t}-W_{t_{i-1}}\right)\right]^{2} d t$.

It is a simple exercise to check that

$$
E\left(\left(e^{u \xi-\frac{1}{2} u^{2}}-1\right) e^{u \xi-\frac{1}{2} u^{2}}-u \xi\right)^{2}=O\left(u^{4}\right), \quad u \rightarrow 0 .
$$

Hence, we can dominate the expectations in the integrals by a quadratic function and obtain that

$$
n E\left(P_{1}^{1 n}-\bar{M}_{1}^{1 n}\right)^{2} \leq \mathrm{const} n \sum_{i=1}^{n-1} \Lambda_{t_{i-1}}\left(\Delta t_{i}\right)^{3} \rightarrow 0, \quad n \rightarrow 0 .
$$

By virtue of the Doob inequality also $n E \sup _{s}\left(P_{s}^{1 n}-\bar{M}_{s}^{1 n}\right)^{2} \rightarrow 0$.

Note that $\bar{M}_{t_{i-1}}^{1 n}=M_{t_{i-1}}^{1 n}$, the process $M^{1 n}$ is constant on the interval $\left[t_{i-1}, t_{i}[\right.$ while

$$
\bar{M}_{s}^{1 n}-\bar{M}_{t_{i-1}}^{1 n}=\sigma^{2} \widehat{C}_{x x}\left(t_{i-1}, S_{t_{i-1}}\right) S_{t_{i-1}}^{2} \int_{t_{i-1}}^{s}\left(W_{t_{i-1}}-W_{t}\right) d W_{t} .
$$

It follows that

$$
n \sup _{s}\left(M_{s}^{1 n}-\bar{M}_{s}^{1 n}\right)^{2}=n \sigma^{4} \max _{i \leq n-1} \widehat{C}_{x x}^{2}\left(t_{i-1}, S_{t_{i-1}}\right) S_{t_{i-1}}^{4} \eta_{i}^{2}
$$

where

$$
\eta_{i}:=\frac{1}{2} \sup _{s \in\left[t_{i-1}, t_{i}\right]}\left|\left(W_{s}-W_{t_{i-1}}\right)^{2}-\left(s-t_{i-i}\right)\right| .
$$

Let $m \in] 1,3 / 2\left[\right.$. Using the elementary inequality $\max _{i}\left|a_{i}\right| \leq \sum_{i}\left|a_{i}\right|$, the independence of increments of the Wiener process from the past, the bound (3.19), and the estimate $E\left|\eta_{i}\right|^{2 m} \leq \kappa\left(\Delta t_{i}\right)^{2 m}$ we obtain that

$n^{m} E \sup _{s}\left(M_{s}^{1 n}-\bar{M}_{s}^{1 n}\right)^{2 m} \leq \kappa n^{m} \sum_{i=1}^{n-1} \frac{\left(\Delta t_{i}\right)^{2 m}}{\left(1-t_{i-1}\right)^{m-1 / 2}}=O\left(n^{m-1}\right), \quad n \rightarrow \infty$.

The sequence $n \sup _{s}\left(M_{s}^{1 n}-\bar{M}_{s}^{1 n}\right)^{2}$ converges to zero in $L^{m}$, hence, in $L^{1}$.

Summarizing, we conclude that $n^{1 / 2}|| R_{1}^{1 M n *} \|_{L^{2}} \rightarrow 0$ as $n \rightarrow \infty$. 
2. The residual process $R^{1 n n}$ is a martingale and by the Doob inequality $E\left(R_{1}^{1 n n *}\right)^{2} \leq 4 E\left(R_{1}^{1 n n}\right)^{2}$. We have:

$$
E\left(R_{1}^{1 n n}\right)^{2}=\int_{t_{n-1}}^{1} E\left(\widehat{C}_{x}\left(t_{n-1}, S_{t_{n-1}}\right)-\widehat{C}_{x}\left(t, S_{t}\right)\right)^{2} S_{t}^{2} d t \leq \kappa_{n}\left(1-t_{n-1}\right)
$$

where $\kappa_{n}$ is the supremum of the integrand over $\left[t_{n-1}, 1\right]$. By virtue of Lemma $3.2 \kappa_{n} \rightarrow 0$. Since $1-t_{n-1} \leq \kappa n^{-1}$ (due to the boundedness of $g^{\prime}$ ), we conclude that $n E\left(R_{1}^{1 n n}\right)^{2} \rightarrow 0$.

3. By the Doob inequality asymptotic analysis of the sequence $R_{1}^{1 t n *}$ can be reduced to that of

$$
R_{1}^{1 t n}=\sum_{i=1}^{n-1} \widehat{C}_{x t}\left(t_{i-1}, S_{t_{i-1}}\right) \int_{t_{i-1}}^{t_{i}}\left(t-t_{i-1}\right) S_{t} d W_{t}
$$

According to $(3.20)$

$$
E \widehat{C}_{x t}^{2}\left(t, S_{t}\right) S_{t}^{2} \leq \kappa \frac{1}{(1-t)^{3 / 2}}
$$

Therefore,

$$
\begin{aligned}
E\left(R_{1}^{1 t n}\right)^{2} & =\sum_{i=1}^{n-1} E \widehat{C}_{x t}^{2}\left(t_{i-1}, S_{t_{i-1}}\right) S_{t_{i-1}}^{2} \int_{t_{i-1}}^{t_{i}}\left(t-t_{i-1}\right)^{2} E\left(S_{t} / S_{t_{i-1}}\right)^{2} d t \\
& \leq \mathrm{const} \sum_{i=1}^{n-1} \frac{\left(\Delta t_{i}\right)^{3}}{\left(1-t_{i-1}\right)^{3 / 2}}=O\left(n^{-3 / 2}\right), \quad n \rightarrow \infty,
\end{aligned}
$$

in virtue of Lemma 4.2. Hence, $E\left(R_{1}^{1 t n}\right)^{2} \rightarrow 0$.

4. Now we estimate the expectation $E\left(\tilde{R}_{1}^{1 n}\right)^{2}$ corresponding to the terminal value of the martingale arising from the remainder term in the Taylor formula for $\widehat{C}_{x}$. We have:

$$
E\left(\tilde{R}_{1}^{1 n}\right)^{2}=\sum_{i=1}^{n-1} \int_{t_{i-1}}^{t_{i}} E\left(\tilde{U}_{t}^{i}\right)^{2} d t
$$

Since $(a+b+c)^{2} \leq 3\left(a^{2}+b^{2}+c^{2}\right)$, it is sufficient to check that each of the following sums converge to zero as $o\left(n^{-1}\right)$ :

$$
\begin{aligned}
\Sigma_{1}^{n} & =\sum_{i=1}^{n-1} \int_{t_{i-1}}^{t_{i}} E \widehat{C}_{x x x}^{2}\left(\tilde{t}_{i-1}, \tilde{S}_{t_{i-1}}\right)\left(S_{t}-S_{t_{i-1}}\right)^{4} S_{t}^{2} d t \\
\Sigma_{2}^{n} & :=\sum_{i=1}^{n-1} \int_{t_{i-1}}^{t_{i}} E \widehat{C}_{x t t}^{2}\left(\tilde{t}_{i-1}, \tilde{S}_{t_{i-1}}\right)\left(t-t_{i-1}\right)^{4} S_{t}^{2} d t \\
\Sigma_{3}^{n} & :=\sum_{i=1}^{n-1} \int_{t_{i-1}}^{t_{i}} E \widehat{C}_{x x t}^{2}\left(\tilde{t}_{i-1}, \tilde{S}_{t_{i-1}}\right)\left(t-t_{i-1}\right)^{2}\left(S_{t}-S_{t_{i-1}}\right)^{2} S_{t}^{2} d t .
\end{aligned}
$$


Using the continuity of the process $S_{t}$ we obtain from the formula (3.11) that

$$
\lim _{t \rightarrow 1} \sup _{r \geq t} \Sigma_{N}\left(S_{r}, \rho_{t}\right)=0 \quad \text { a.s. }
$$

Applying Lemma 3.6 we infer that for any $\varepsilon>0, m \geq 1$, there exists $a \in] 0,1[$ such that

$$
E\left|\widehat{C}_{x x x}\left(\tilde{t}_{i-1}, \tilde{S}_{t_{i-1}}\right)\right|^{2 m} \leq \varepsilon \frac{1}{\left(1-t_{i}\right)^{2 m}}
$$

for every $t_{i-1} \geq a$. For $t_{i-1}<a$ the above expectation is bounded by a constant which does not on $n$.

Let $\xi \sim N(0,1)$ and let $b \in[0,1]$. Using the elementary bound

$$
\left|e^{b x}-1\right| \leq b\left(e^{|x|}-1\right)
$$

which follows from the Taylor expansion, we obtain, for $m \geq 1$, the estimate

$$
E\left(e^{u \sigma \xi-(1 / 2) \sigma^{2} u^{2}}-1\right)^{2 m} \leq \kappa u^{2 m}
$$

where the constant $\kappa$ depends on $m$ and $\sigma$. Applying the Cauchy-Schwarz inequality and this estimate we get that

$$
E\left(S_{t}-S_{t_{i-1}}\right)^{2 m} S_{t}^{p} \leq \kappa\left(t-t_{i-1}\right)^{m}
$$

Manipulating again with the Cauchy-Schwarz inequality we obtain with the help of the above bounds that

$$
\Sigma_{1}^{n} \leq \kappa \sum_{t_{i-1}<a}\left(\Delta t_{i}\right)^{3}+\kappa \varepsilon \sum_{i=1}^{n-1} \frac{\left(\Delta t_{i}\right)^{3}}{\left(1-t_{i}\right)^{2}}
$$

The first sum in the right-hand side is of order $O\left(n^{-2}\right)$. According to Lemma 4.2 the second one is of order $O\left(n^{-1}\right)$. Since $\varepsilon>0$ is arbitrary, it follows that $\lim _{n} n \Sigma_{1}^{n}=0$.

Similarly to the bound (6.1) but referring now to Lemma 3.7, we can establish that for any $\varepsilon>0$ there is a threshold $a \in] 0,1]$ such that for any $t_{i-1} \geq a$ the following inequalities hold:

$$
E\left|\widehat{C}_{x x t}\left(\tilde{t}_{i-1}, \tilde{S}_{t_{i-1}}\right)\right|^{2 m} \leq \varepsilon \frac{1}{\left(1-t_{i}\right)^{3 m}}
$$

and

$$
E\left|\widehat{C}_{x t t}\left(\tilde{t}_{i-1}, \tilde{S}_{t_{i-1}}\right)\right|^{2 m} \leq \varepsilon \frac{1}{\left(1-t_{i}\right)^{4 m}} .
$$

With these bounds we prove, making obvious changes in arguments, that $\lim _{n} n \Sigma_{2}^{n}=0$ and $\lim _{n} n \Sigma_{3}^{n}=0$. Thus, $n E\left(\tilde{R}_{1}^{1 n *}\right)^{2} \rightarrow 0$. 


\section{Analysis of the Residual $R^{2 n}$}

Now we give a proof of Proposition 2.4.

1. Put (for $s<1$ )

$$
\bar{P}_{s}^{2 n}=k_{0} \frac{1}{\sqrt{n}} \sum_{t_{i} \leq s} \widehat{C}_{x x}\left(t_{i-1}, S_{t_{i-1}}\right) S_{t_{i-1}}^{2}\left[E\left|S_{t_{i}} / S_{t_{i-1}}-1\right|-\left|S_{t_{i}} / S_{t_{i-1}}-1\right|\right] .
$$

The processes $P^{2 n}, \bar{P}^{2 n}$, and $M^{2 n}$ have piecewise constant trajectories jumping at the moments $t_{i}, i \leq n-1$. Thus,

$$
|| \sup _{s}\left|P_{s}^{2 n}-M_{s}^{2 n}\right|||_{L^{2}} \leq\left.|| \sup _{i}\left|P_{t_{i}}^{2 n}-\bar{P}_{t_{i}}^{2 n}\right|\right|_{L^{2}}+|| \sup _{i}\left|\bar{P}_{t_{i}}^{2 n}-M_{t_{i}}^{2 n}\right|||_{L^{2}} .
$$

We have:

$$
n^{1 / 2}\left\|\sup _{i}\left|P_{t_{i}}^{2 n}-\bar{P}_{t_{i}}^{2 n}\right|\right\|_{L^{2}} \leq k_{0} \sum_{i=1}^{n-1} \Lambda_{t_{i-1}}^{1 / 2} B_{i}
$$

where

$$
B_{i}:=\left|\sigma \sqrt{2 / \pi} \sqrt{n f^{\prime}\left(t_{i-1}\right)} \Delta t_{i}-E\right| S_{t_{i}} / S_{t_{i-1}}-1||
$$

Using the Taylor formula it is easy to verify that for $u>0$

$$
E\left|e^{u \xi-\frac{1}{2} u^{2}}-1\right|=2[\Phi(u / 2)-\Phi(-u / 2)]=\sqrt{2 / \pi} u+O\left(u^{3}\right), \quad u \rightarrow 0,
$$

It follows that

$$
B_{i}=\sigma \sqrt{2 / \pi}\left(\Delta t_{i}\right)^{1 / 2}\left|\sqrt{n f^{\prime}\left(t_{i-1}\right) \Delta t_{i}}-1\right|+O\left(\left(\Delta t_{i}\right)^{3 / 2}\right) .
$$

By the Taylor formula

$$
\Delta t_{i}=g(i / n)-g((i-1) / n)=g^{\prime}((i-1) / n) \frac{1}{n}+\frac{1}{2} g^{\prime \prime}\left(y_{i}\right) \frac{1}{n^{2}},
$$

where the point $y_{i} \in[(i-1) / n, i / n]$. Since $f$ is the inverse of $g$ we have $f^{\prime}\left(t_{i-1}\right)=1 / g^{\prime}((i-1) / n)$. Using these identities and the elementary inequality $|\sqrt{1+a}-1| \leq|a|$ for $a \geq-1$ we obtain that

$$
B_{i} \leq \mathrm{const} \frac{\left|g^{\prime \prime}\left(y_{i}\right)\right|}{g^{\prime}((i-1) / n)}\left(\Delta t_{i}\right)^{1 / 2} \frac{1}{n}+O\left(\left(\Delta t_{i}\right)^{3 / 2}\right)
$$

Fix $\varepsilon \in] 0,1 / 4\left[\right.$. Substituting the finite increments formula $\Delta t_{i}=g^{\prime}\left(x_{i}\right) / n$ with an intermediate point $x_{i}$ in $[(i-1) / n, i / n]$, we infer that

$$
B_{i} \leq \operatorname{const} a_{n} \frac{g^{\prime}\left(x_{i}\right)}{[1-g((i-1) / n)]^{3 / 4-\varepsilon}} \frac{1}{n}+O\left(\left(\Delta t_{i}\right)^{3 / 2}\right) .
$$

where

$$
a_{n}=\frac{1}{n^{1 / 2}} \sup _{i \leq n-1} \sup _{x_{i}, y_{i}} \frac{\left|g^{\prime \prime}\left(y_{i}\right)\right|[1-g((i-1) / n)]^{3 / 4-\varepsilon}}{g^{\prime}((i-1) / n)\left(g^{\prime}\left(x_{i}\right)\right)^{1 / 2}} .
$$


Recall that

$$
\sum_{i=1}^{n-1} \frac{g^{\prime}\left(x_{i}\right)}{[1-g((i-1) / n)]^{1-\varepsilon}} \frac{1}{n} \rightarrow \int_{0}^{1} \frac{d g(t)}{[1-g(t)]^{1-\varepsilon}}=\int_{0}^{1} \frac{d t}{(1-t)^{1-\varepsilon}}<\infty
$$

and $a_{n} \rightarrow 0$ under each of our assumptions. These observations lead to the conclusion that

$$
\sum_{i=1}^{n-1} \Lambda_{t_{i-1}}^{1 / 2} B_{i} \rightarrow 0
$$

Noticing that

$$
E\left(\left|e^{u \xi-\frac{1}{2} u^{2}}-1\right|-u|\xi|\right)^{2}=O\left(u^{4}\right), \quad u \rightarrow 0,
$$

we infer that

$E\left[\left(E\left|S_{t_{i}} / S_{t_{i-1}}-1\right|-\left|S_{t_{i}} / S_{t_{i-1}}-1\right|\right)-\sigma\left(E\left|\Delta W_{t_{i}}\right|-\left|\Delta W_{t_{i}}\right|\right)\right]^{2}=O\left(\left(\Delta t_{i}\right)^{2}\right)$.

Applying Lemma 4.1 and the Doob inequality to the discrete-time squareintegrable martingale $\left(\bar{P}_{t_{i}}^{2 n}-M_{t_{i}}^{2 n}, \mathcal{F}_{t_{i}}\right)$, we get that

$$
n \operatorname{Esup}_{i}\left|\bar{P}_{t_{i}}^{2 n}-M_{t_{i}}^{2 n}\right|^{2} \leq \mathrm{const} \sum_{i=1}^{n-1} \Lambda_{t_{i-1}}\left(\Delta t_{i}\right)^{2} \rightarrow 0, \quad n \rightarrow \infty .
$$

We conclude that $n^{1 / 2}\left\|R_{1}^{2 M n *}\right\|_{L^{2}} \rightarrow 0$ as $n \rightarrow \infty$.

2. Noting that $\left\|S_{t}^{2} \widehat{C}_{x x}\left(t, S_{t}\right)\right\|_{L^{2}}=\Lambda_{t}^{1 / 2}$, we have:

$$
\left\|R_{1}^{2 n n}\right\|_{L^{2}} \leq \int_{t_{n-1}}^{1} \Lambda_{t}^{1 / 2} \sqrt{f^{\prime}(t)} d t \leq\left(\int_{t_{n-1}}^{1} \Lambda_{t} d t\right)^{1 / 2}\left(1-f\left(t_{n-1}\right)\right)^{1 / 2} .
$$

Since $f\left(t_{n-1}\right)=f(g((n-1) / n))=1-1 / n$ and the function $\Lambda$ is integrable, it follows that $n E\left(R_{1}^{2 n n}\right)^{2} \rightarrow 0$.

3. The process $R^{21 n}$ describes the error in approximation of an integral by the Riemann sums. To analyze the approximation rate we need the following auxiliary result.

Lemma 7.1 Let $X=\left(X_{t}\right)_{t \in[0, T]}$ be a process with

$$
d X_{t}=\mu_{t} d t+\vartheta_{t} d W_{t}, \quad X_{0}=0,
$$

where $\mu=\left(\mu_{t}\right)_{t \in[0, T]}$ and $\vartheta=\left(\vartheta_{t}\right)_{t \in[0, T]}$ are predictable processes such that

$$
\int_{0}^{T}\left(\left|\mu_{t}\right|+\vartheta_{t}^{2}\right) d t<\infty
$$

Let $X_{t}^{n}:=\sum_{i=1}^{n} X_{t_{i-1}} I_{\left.t_{i-1}, t_{i}\right]}(t)$. Then

$$
\begin{aligned}
E \sup _{s \in[0, T]}\left(\int_{0}^{s}\left(X_{t}-X_{t}^{n}\right) d t\right)^{2} \leq & 8 \int_{0}^{T} \sum_{i=1}^{n}\left(t_{i}-u\right)^{2} I_{] t_{i-1}, t_{i}\right]}(u) E \vartheta_{u}^{2} d u \\
& +2\left(\int_{0}^{T} \sum_{i=1}^{n}\left(t_{i}-u\right) I_{] t_{i-1}, t_{i}\right]}(u)\left(E \mu_{u}^{2}\right)^{1 / 2} d u\right)^{2} .
\end{aligned}
$$


Proof. It is sufficient to work assuming that the right-hand side of the inequality is finite. Having in mind that $(a+b)^{2} \leq 2 a^{2}+2 b^{2}$, we may consider separately the cases where one of the coefficients is zero. Let us start with the case where $\mu=0$. For $v \in\left[t_{i-1}, t_{i}\right.$ [ we have, using the stochastic Fubini theorem:

$$
\int_{t_{i-1}}^{v}\left(X_{t}-X_{t_{i-1}}\right) d t=\int_{t_{i-1}}^{v} \int_{t_{i-1}}^{v} \vartheta_{u} I_{] t_{i-1}, t\right]}(u) d W_{u} d t=\int_{t_{i-1}}^{v}(v-u) \vartheta_{u} d W_{u}
$$

Thus,

$$
\int_{0}^{s}\left(X_{t}-X_{t}^{n}\right) d t=\int_{0}^{s} \sum_{i=1}^{n}\left(t_{i}-u\right) I_{] t_{i-1}, t_{i}\right]}(u) \vartheta_{u} d W_{u}
$$

The right-hand side is a local martingale and we obtain from the Doob inequality

$$
E \sup _{s \in[0, T]}\left(\int_{0}^{s}\left(X_{t}-X_{t}^{n}\right) d t\right)^{2} \leq 4 \int_{0}^{T} \sum_{i=1}^{n}\left(t_{i}-u\right)^{2} I_{] t_{i-1}, t_{i}\right]}(u) E \vartheta_{u}^{2} d u
$$

In the case where $\vartheta=0$ we have, this time by the ordinary Fubini theorem, that

$$
\int_{t_{i-1}}^{v}\left(X_{t}-X_{t_{i-1}}\right) d t=\int_{t_{i-1}}^{v}(v-u) \mu_{u} d u, \quad v \in\left[t_{i-1}, t_{i}[,\right.
$$

and this representation allows us to transform the squared process of interest to the following form:

$$
\int_{0}^{s} \int_{0}^{s} \sum_{i, j=1}^{n}\left(t_{i}-u\right)\left(t_{j}-r\right) I_{] t_{i-1}, t_{i}\right]}(u) I_{] t_{j-1}, t_{j}\right]}(r) \mu_{u} \mu_{r} d u d r .
$$

Its expectation can be dominated by

$$
\int_{0}^{s} \int_{0}^{s} \sum_{i, j=1}^{n}\left(t_{i}-u\right)\left(t_{j}-r\right) I_{] t_{i-1}, t_{i}\right]}(u) I_{] t_{j-1}, t_{j}\right]}(r) E\left|\mu_{u} \mu_{r}\right| d u d r .
$$

Using the Cauchy-Schwarz inequality $E\left|\mu_{u} \mu_{r}\right| \leq\left(E \mu_{u}^{2}\right)^{1 / 2}\left(E \mu_{r}^{2}\right)^{1 / 2}$ and once again by the Fubini theorem we obtain the needed bound.

Let $X_{t}:=S_{t}^{2} \widehat{C}_{x x}\left(t, S_{t}\right) \sqrt{f^{\prime}(t)} I_{\left[0, t_{n-1}\right]}$. Then

$$
R_{s}^{21 n}=Y_{s}^{n}+\int_{0}^{s} X_{t}^{n} d t-\sum_{t_{i} \leq s} \widehat{C}_{x x}\left(t_{i-1}, S_{t_{i-1}}\right) S_{t_{i-1}}^{2} \sqrt{f^{\prime}\left(t_{i-1}\right)} \Delta t_{i}, \quad s<1
$$

where

$$
Y_{s}^{n}:=\int_{0}^{s}\left(X_{t}-X_{t}^{n}\right) d t
$$


The process $X$ on the interval $\left[0, t_{n-1}\right]$ admits the representation of the above lemma with the coefficients

$$
\begin{aligned}
\vartheta_{t}= & {\left[2 S_{t} \widehat{C}_{x x}\left(t, S_{t}\right)+S_{t}^{2} \widehat{C}_{x x x}\left(t, S_{t}\right)\right] \sqrt{f^{\prime}(t)} \sigma S_{t}, } \\
\mu_{t}= & \frac{1}{2}\left[2 \widehat{C}_{x x}\left(t, S_{t}\right)+4 S_{t} \widehat{C}_{x x x}\left(t, S_{t}\right)+S_{t}^{2} \widehat{C}_{x x x x}\left(t, S_{t}\right)\right] \sqrt{f^{\prime}(t)} \sigma^{2} S_{t}^{2} \\
& +\frac{1}{2} S_{t}^{2} \widehat{C}_{x x}\left(t, S_{t}\right) \frac{f^{\prime \prime}(t)}{\sqrt{f^{\prime}(t)}}+S_{t}^{2} \widehat{C}_{x x t}\left(t, S_{t}\right) \sqrt{f^{\prime}(t)} .
\end{aligned}
$$

In the case where $g^{\prime}$ is bounded away from zero (hence, $f^{\prime}$ is bounded), the estimates (3.16) and (3.21) imply that $E \vartheta_{t}^{2} \leq \kappa /(1-t)^{3 / 2}$. If also $f^{\prime \prime}$ is bounded, then the estimates (3.16) and (3.21) - (3.23) ensure that $E \mu_{t}^{2} \leq$ $\kappa /(1-t)^{5 / 2}$.

Applying the lemma we have:

$$
E \sup _{s \in[0,1]}\left|Y_{s}^{n}\right|^{2} \leq \kappa \sum_{i=1}^{n-1} \frac{\left(\Delta t_{i}\right)^{3}}{\left(1-t_{i}\right)^{3 / 2}}+\kappa\left(\sum_{i=1}^{n-1} \frac{\left(\Delta t_{i}\right)^{2}}{\left(1-t_{i}\right)^{5 / 4}}\right)^{2} .
$$

According to Lemma 4.2 the right-hand side is $O\left(n^{-3 / 2}\right)$ as $n \rightarrow \infty$.

In the case where $g(t)=1-(1-t)^{\beta}, \beta>1$, we obtain in the same way that $E \vartheta_{t}^{2} \leq \kappa /(1-t)^{5 / 2-1 / \beta}, E \mu_{t}^{2} \leq \kappa /(1-t)^{7 / 2-1 / \beta}$, and

$$
E \sup _{s \in[0,1]}\left|Y_{s}^{n}\right|^{2} \leq \kappa \sum_{i=1}^{n-1} \frac{\left(\Delta t_{i}\right)^{3}}{\left(1-t_{i}\right)^{5 / 2-1 / \beta}}+\kappa\left(\sum_{i=1}^{n-1} \frac{\left(\Delta t_{i}\right)^{2}}{\left(1-t_{i}\right)^{7 / 4-1 /(2 \beta)}}\right)^{2} .
$$

By Lemma 4.2 the first sum in the right-hand side can be of order $O\left(n^{-2}\right)$, $O\left(n^{-2} \ln n\right)$, or $O\left(n^{-(\beta / 2+1)}\right)$, that is $o\left(n^{-1}\right)$ as $n \rightarrow \infty$. The second sum can be $O\left(n^{-1}\right), O\left(n^{-1} \ln n\right)$, or $O\left(n^{-(\beta / 4+1 / 2)}\right)$, i.e. $o\left(n^{-1 / 2}\right)$. In all cases $n E \sup _{s}\left|Y_{s}^{n}\right|^{2} \rightarrow 0$.

The process $R^{21 n}-Y^{n}$ vanishes in the revision dates and

$$
\sup _{s \in[0,1]}\left|R_{s}^{21 n}-Y_{s}^{n}\right| \leq \kappa \max _{i \leq n-1} \int_{t_{i-1}}^{t_{i}} \widehat{C}_{x x}\left(t, S_{t}\right) S_{t}^{2} \sqrt{f^{\prime}(t)} d t
$$

By the Cauchy-Schwarz inequality

$$
\int_{t_{i-1}}^{t_{i}} \widehat{C}_{x x}\left(t, S_{t}\right) S_{t}^{2} \sqrt{f^{\prime}(t)} d t \leq\left(\int_{t_{i-1}}^{t_{i}} \widehat{C}_{x x}^{2}\left(t, S_{t}\right) S_{t}^{4} d t\right)^{1 / 2}\left(\int_{t_{i-1}}^{t_{i}} f^{\prime}(t) d t\right)^{1 / 2}
$$

Note that the second integral in the right-hand side is equal to $1 / n$.

Using the bound $\max _{i}\left|a_{i}\right| \leq \sum_{i}\left|a_{i}\right|$, the Jensen inequality, and the estimate (3.19) we obtain from here that for $m \geq 3 / 2$

$$
n^{m} E \sup _{s \in[0,1]}\left|R_{s}^{21 n}-Y_{s}^{n}\right|^{2 m} \leq \kappa E\left(\sum_{i \leq n-1} \int_{t_{i-1}}^{t_{i}} \widehat{C}_{x x}^{2}\left(t, S_{t}\right) S_{t}^{4} d t\right)^{m}
$$




$$
\begin{aligned}
& \leq \kappa \sum_{i \leq n-1}\left(\Delta t_{i}\right)^{m-1} \int_{t_{i-1}}^{t_{i}} E \widehat{C}_{x x}^{2 m}\left(t, S_{t}\right) S_{t}^{4 m} d t \\
& \leq \kappa \sum_{i \leq n-1} \frac{\left(\Delta t_{i}\right)^{m}}{\left(1-t_{i}\right)^{m-1 / 2}}=O\left(n^{-1 / 2}\right)
\end{aligned}
$$

by virtue of Lemma 4.2. That is, $n \sup _{s \in[0,1]}\left|R_{s}^{21 n}-Y_{s}^{n}\right|^{2}$ tends to zero in $L^{m}$ and, hence, in $L^{1}$.

4. The residual processes $R_{s}^{22 n}$ have piecewise constant trajectories and the analysis of the asymptotic behavior is reduced to the discrete-time scheme.

Let

$$
\xi_{i}^{n}:=\left(S_{t_{i}} / S_{t_{i-1}}-1\right)^{2} \operatorname{sign}\left(S_{t_{i}} / S_{t_{i-1}}-1\right),
$$

$\Delta M_{i}^{n}:=\xi_{i}^{n}-E \xi_{i}^{n}$, and $X_{i}^{n}:=\widehat{C}_{x x}\left(t_{i-1}, S_{t_{i-1}}\right) S_{t_{i-1}}^{2}$. With this notation we have the representation

$$
n^{1 / 2} R_{t_{k}}^{22 n}=X^{n} \cdot M_{k}^{n}+A_{k}^{n}, \quad k \leq n-1,
$$

where

$$
A_{k}^{n}:=\sum_{i \leq k} X_{i}^{n} E \xi_{i}^{n} .
$$

Note that

$$
E\left(e^{u \xi-\frac{1}{2} u^{2}}-1\right)^{4}=O\left(u^{4}\right), \quad u \rightarrow 0 .
$$

Applying the Doob inequality and Lemma 4.1 we obtain that

$$
\begin{aligned}
E \sup _{i \leq n-1}\left(X^{n} \cdot M_{n-1}^{n}\right)^{2} \leq 4 E\left(X^{n} \cdot M_{n-1}^{n}\right)^{2} & \leq 4 \sum_{i \leq n-1} \Lambda_{t_{i-1}}\left(S_{t_{i}} / S_{t_{i-1}}-1\right)^{4} \\
& \leq \kappa \sum_{i \leq n-1} \frac{\left(\Delta t_{i}\right)^{2}}{\left(1-t_{i-1}\right)^{1 / 2}}=O\left(n^{-1}\right)
\end{aligned}
$$

according to Lemma 4.2 .

By virtue of Lemma 8.1 given below in the section on asymptotics of Gaussian integrals for sufficiently large $n$ we have the inequalities

$$
0 \leq E \xi_{i}^{n} \leq \kappa\left(\Delta t_{i}\right)^{3 / 2}
$$

implying that the discrete-time process $A$ is increasing and

$$
\begin{aligned}
\left\|A_{n-1}^{n}\right\|_{L^{2}} \leq \sum_{i \leq n-1}\left\|X_{i}^{n}\right\|_{L^{2}} E \xi_{i}^{n} & \leq \kappa \sum_{i \leq n-1} \Lambda_{t_{i-1}}^{1 / 2}\left(\Delta t_{i}\right)^{3 / 2} \\
& \leq \kappa \sum_{i \leq n-1} \frac{\left(\Delta t_{i}\right)^{3 / 2}}{\left(1-t_{i-1}\right)^{1 / 4}}=O\left(n^{-1}\right)
\end{aligned}
$$

again according to Lemma 4.2

It follows that $n E\left(R_{1}^{22 n *}\right)^{2} \rightarrow 0$.

5. We verify now that $n E\left(R_{1}^{23 n *}\right)^{2} \rightarrow 0$. Recall that

$$
E\left(S_{t_{i}}-S_{t_{i-1}}\right)^{2 m} \leq c_{m}\left(\Delta t_{i}\right)^{m} .
$$


Using (3.20) we obtain the bound

$$
E \widehat{C}_{x t}^{2}\left(t_{i-1}, S_{t_{i-1}}\right)\left(\Delta t_{i}\right)^{2}\left(S_{t_{i}}-S_{t_{i-1}}\right)^{2} \leq \kappa \frac{\left(\Delta t_{i}\right)^{3}}{\left(1-t_{i-1}\right)^{3 / 2}} .
$$

To estimate the terms coming from the residual term of the Taylor expansion we use the Cauchy-Schwarz inequality and the bounds (3.5), (3.8), (3.9). This yields in the following:

$$
\begin{aligned}
E \widehat{C}_{x x x}^{2}\left(\tilde{t}_{i-1}, \tilde{S}_{t_{i-1}}\right)\left(S_{t_{i}}-S_{t_{i-1}}\right)^{6} & \leq \kappa \frac{\left(\Delta t_{i}\right)^{3}}{\left(1-t_{i}\right)^{2}}, \\
E \widehat{C}_{x x t}^{2}\left(\tilde{t}_{i-1}, \tilde{S}_{t_{i-1}}\right)\left(S_{t_{i}}-S_{t_{i-1}}\right)^{4}\left(\Delta t_{i}\right)^{2} & \leq \kappa \frac{\left(\Delta t_{i}\right)^{4}}{\left(1-t_{i}\right)^{3}}, \\
E \widehat{C}_{x t t}^{2}\left(\tilde{t}_{i-1}, \tilde{S}_{t_{i-1}}\right)\left(\Delta t_{i}\right)^{4}\left(S_{t_{i}}-S_{t_{i-1}}\right)^{2} & \leq \kappa \frac{\left(\Delta t_{i}\right)^{5}}{\left(1-t_{i}\right)^{4}} .
\end{aligned}
$$

Obviously,

$$
n^{1 / 2}\left\|R_{1}^{23 n *}\right\|_{L^{2}} \leq \sum_{i \leq n-1}\left\|[\ldots]_{i}\left(S_{t_{i}}-S_{t_{i-1}}\right)\right\|_{L^{2}}
$$

where $[\ldots]_{i}$ is defined in $(2.3)$. Taking into account that $\widehat{C}_{x x}(t, x) \geq 0$ and using the inequality ||$a|-| b|| \leq|a-b|$ we can write that

$$
\left\|[\ldots]_{i}\left(S_{t_{i}}-S_{t_{i-1}}\right)\right\|_{L^{2}} \leq \kappa\left(\left\|\widehat{C}_{x t}\left(t_{i-1}, S_{t_{i-1}}\right)\left(t_{i}-t_{i-1}\right)\left(S_{t_{i}}-S_{t_{i-1}}\right)\right\|_{L^{2}}+\ldots\right)
$$

where we denote by dots the $L^{2}$-norms of the residual term in the first order Taylor expansion of the difference $\widehat{C}_{x}\left(t_{i}, S_{t_{i}}\right)-\widehat{C}_{x}\left(t_{i-1}, S_{t_{i-1}}\right)$. Summing up and using the above estimates we conclude, applying Lemma 4.2 , that the right-hand side of the above inequality tends to zero as $n \rightarrow \infty$ and we conclude.

6. It remains to check that $n E\left(R_{1}^{24 n}\right)^{2} \rightarrow 0$ and this happens to be the most delicate part of the proof. Again the analysis can be reduced to the discrete-time case. We note that

$$
n E\left(R_{1}^{24 n}\right)^{2} \leq \sum_{i \leq n-1} E S_{t_{i-1}}^{2}[\ldots]_{i}^{2}+2 \sum_{i<j} E\left|S_{t_{i-1}}[\ldots]_{i} S_{t_{j-1}}[\ldots]_{j}\right|
$$

The estimation of the first sum is rather straightforward. Applying the Ito formula to the function $\widehat{C}_{x}(t, x)$ and using the positivity of $\widehat{C}_{x x}(t, x)$ and the inequality ||$a|-| b|| \leq|a-b|$ we dominate the absolute value of random variable denoted by $[\ldots]_{i}$, see the formula $(2.3)$, by the absolute value of

$\int_{t_{i-1}}^{t_{i}}\left(\widehat{C}_{x x}\left(t_{i-1}, S_{t_{i-1}}\right)-\widehat{C}_{x x}\left(t, S_{t}\right)\right) d S_{t}-\int_{t_{i-1}}^{t_{i}}\left(\widehat{C}_{x t}\left(t, S_{t}\right)+\frac{\sigma^{2}}{2} S_{t}^{2} \widehat{C}_{x x x}\left(t, S_{t}\right)\right) d t$. 
We check that

$$
\begin{gathered}
\sum_{i=1}^{n-1} E S_{t_{i-1}}^{2} \int_{t_{i-1}}^{t_{i}}\left(\widehat{C}_{x x}\left(t_{i-1}, S_{t_{i-1}}\right)-\widehat{C}_{x x}\left(t, S_{t}\right)\right)^{2} S_{t}^{2} d t=O\left(n^{-1 / 4}\right), \\
\sum_{i=1}^{n-1} \Delta t_{i} E S_{t_{i-1}}^{2} \int_{t_{i-1}}^{t_{i}}\left(\widehat{C}_{x t}^{2}\left(t, S_{t}\right)+S_{t}^{4} \widehat{C}_{x x x}^{2}\left(t, S_{t}\right)\right) d t=O\left(n^{-1 / 2}\right) .
\end{gathered}
$$

A generic term of the first sum is dominated by

$$
\Delta t_{i} E \sup _{t \leq 1} S_{t}^{4} \sup _{t_{i-1} \leq t \leq t_{i}}\left(\widehat{C}_{x x}\left(t, S_{t}\right)-\widehat{C}_{x x}\left(t_{i-1}, S_{t_{i-1}}\right)\right)^{2}
$$

The Cauchy-Schwarz inequality allows us to separate the terms under the sign of expectation and reduce the problem to the estimation of the forth power of the difference $\widehat{C}_{x x}\left(t, S_{t}\right)-\widehat{C}_{x x}\left(t_{i-1}, S_{t_{i-1}}\right)$. The Ito formula transforms this difference into the sum of a stochastic integral and an ordinary integral. Using consecutively the Burkholder and Cauchy-Schwarz inequalities and the bound (3.21) we have:

$$
\begin{aligned}
E \sup _{t \in\left[t_{i-1}, t_{i}\right]}\left[\int_{t_{i-1}}^{t} \widehat{C}_{x x x}\left(u, S_{u}\right) S_{u} d S_{u}\right]^{4} & \leq c_{4} E\left[\int_{t_{i-1}}^{t_{i}} \widehat{C}_{x x x}^{2}\left(u, S_{u}\right) S_{u}^{4} d u\right]^{2} \\
& \leq c_{4} \Delta t_{i} E \int_{t_{i-1}}^{t_{i}} \widehat{C}_{x x x}^{4}\left(u, S_{u}\right) S_{u}^{8} d u \\
& \leq \kappa \frac{\left(\Delta t_{i}\right)^{2}}{\left(1-t_{i}\right)^{7 / 2}}
\end{aligned}
$$

To estimate the ordinary integral we use the Jensen inequality for $f(x)=x^{4}$ and the bounds (3.22) and (3.23) and get that

$$
E \sup _{t \in\left[t_{i-1}, t_{i}\right]}\left[\int_{t_{i-1}}^{t}\left(\widehat{C}_{x x t}\left(u, S_{u}\right)+\frac{1}{2} \sigma^{2} S_{u}^{2} \widehat{C}_{x x x x}\left(u, S_{u}\right)\right) d u\right]^{4} \leq \kappa \frac{\left(\Delta t_{i}\right)^{4}}{\left(1-t_{i}\right)^{11 / 2}} .
$$

Using these estimates we obtain that the sum in (7.1) is dominated, up to a multiplicative constant, by

$$
\sum_{i=1}^{n-1}\left[\frac{\left(\Delta t_{i}\right)^{2}}{\left(1-t_{i}\right)^{7 / 4}}+\frac{\left(\Delta t_{i}\right)^{3}}{\left(1-t_{i}\right)^{11 / 4}}\right]
$$

and the claimed asymptotics follows from Lemma 4.2 .

Similar arguments, but using the inequalities (3.20) and (3.21), give us the second asymptotic formula.

From the same estimates we obtain that

$$
\sum_{i=1}^{n-1}\left(E S_{t_{i-1}}^{2}[\ldots]_{i}^{2}\right)^{1 / 2} \leq \kappa \sum_{i=1}^{n-1} \frac{\Delta t_{i}}{\left(1-t_{i}\right)^{7 / 8}}+\kappa \sum_{i=1}^{n-1} \frac{\left(\Delta t_{i}\right)^{3 / 2}}{\left(1-t_{i}\right)^{11 / 8}} .
$$


The second sum in the right-hand side converges to zero while for the first one we can say only that it is dominated by a convergent integral. Using this observation we conclude that the sum of expectations of cross terms over indices $i, j$ with $i<j$ and $t_{j}>a$ also can be done arbitrary small by choosing $a$ sufficiently close to one.

Unexpectedly, the most difficult part of the proof is in establishing the convergence to zero of the sum of cross terms corresponding to the dates of revisions before $a<1$, i.e. bounded away from the singularity.

To formulate the claim we introduce "reasonable" notations. Put

$$
\begin{aligned}
\alpha_{i} & :=\widehat{C}_{x x}\left(S_{t_{i-1}}, t_{i-1}\right) S_{t_{i-1}}^{2}\left(\frac{S_{t_{i}}}{S_{t_{i-1}}}-1\right), \\
\beta_{i} & :=S_{t_{i-1}} \widehat{C}_{x t}\left(S_{t_{i-1}}, t_{i-1}\right) \Delta t_{i}+\frac{1}{2} S_{t_{i-1}}^{3} \widehat{C}_{x x x}\left(S_{t_{i-1}}, t_{i-1}\right)\left(\frac{S_{t_{i}}}{S_{t_{i-1}}}-1\right)^{2},
\end{aligned}
$$

$\gamma_{i}:=\left|\alpha_{i}+\beta_{i}\right|-\left|\alpha_{i}\right|$. Let us define also the random variable $\chi_{i}:=\operatorname{sign}\left(\alpha_{i} \beta_{i}\right)$ and the set $A_{i}:=\left\{\left|\beta_{i}\right|<\left|\alpha_{i}\right|\right\}$.

Now we have the identity

$$
S_{t_{i-1}}[\ldots]_{i}=-\gamma_{i}+\zeta_{i}
$$

where the expression $[\ldots]_{i}$ given in $(2.3)$ and

$$
\zeta_{i}:=|\alpha+\beta|-\left|\widehat{C}_{x}\left(t_{i}, S_{t_{i}}\right)-\widehat{C}_{x}\left(t_{i-1}, S_{t_{i-1}}\right)\right| S_{t_{i-1}}
$$

Using the first order Taylor expansion of $\widehat{C}_{x}(t, x)$ and estimates of the higher order derivatives we get the bound

$$
\left|\zeta_{i}\right| \leq \kappa_{a} \eta\left(\left|\Delta S_{t_{i}}\right|^{2}+\left|\Delta S_{t_{i}}\right| \Delta t_{i}+\left(\Delta t_{i}\right)^{2}\right)\left|\Delta S_{t_{i}}\right|
$$

where $\eta$ is a power of the random variable $\sup _{t<1} S_{t}$ having moments of any order. It follows that

$$
E\left|\zeta_{i}\right|^{2} \leq \kappa\left(\Delta t_{i}\right)^{3}
$$

For By the Cauchy-Schwarz inequality we infer from here that

$$
\sum_{t_{i} \leq a, t_{j} \leq a} E\left|\zeta_{i} \zeta_{j}\right| \leq \sum_{t_{i} \leq a} \cdot E\left|\zeta_{i}\right|^{2}=O\left(n^{-2}\right), \quad n \rightarrow \infty .
$$

For $\gamma_{i}$ the straightforward estimate is worse: $E\left|\gamma_{i}\right|^{2} \leq \kappa \Delta t_{i}$. Nevertheless,

$$
\sum_{t_{i} \leq a, t_{j} \leq a} E\left|\gamma_{i} \zeta_{j}\right| \leq\left(\sum_{t_{i} \leq a} E\left|\gamma_{i}\right|^{2}\right)^{1 / 2}\left(\sum_{t_{i} \leq a} E\left|\zeta_{i}\right|^{2}\right)^{1 / 2}=O\left(n^{-1}\right), \quad n \rightarrow \infty .
$$

The assertion needed to conclude is the lemma below. It is based on asymptotic analysis of expectations of some Gaussian integrals which are given in the next section and the following identities:

$$
\begin{aligned}
|\alpha+\beta|-|\alpha| & =|\beta| \chi I_{A}+|\beta| I_{\{\chi>0\}} I_{A^{c}}+(|\beta|-2|\alpha|) I_{\{\chi \leq 0\}} I_{A^{c}} \\
& =|\beta| \chi+2(|\beta|-|\alpha|) I_{\{\chi \leq 0\}} I_{A^{c}}-|\beta| I_{\{\chi=0\}} I_{A^{c}}
\end{aligned}
$$

where $\alpha, \beta$ are arbitrary random variables, $\chi:=\operatorname{sign}(\alpha \beta), A:=\{|\beta|<|\alpha|\}$. 
Lemma 7.2 For every fixed $a \in] 0,1[$

$$
\left|\sum_{i<j, t_{j} \leq a} E \gamma_{i} \gamma_{j}\right|=o(1), \quad n \rightarrow \infty .
$$

Proof. The routine estimation $\left|E \gamma_{i} \gamma_{j}\right| \leq E\left|\gamma_{i}\right|\left|\gamma_{j}\right|$ does not work in our case. But for $i<j$

$$
\left|E \gamma_{i} \gamma_{j}\right|=\left|E\left(\gamma_{i} E\left(\gamma_{j} \mid \mathcal{F}_{t_{j-1}}\right)\right)\right| \leq E\left(\left|\gamma_{i}\right|\left|E\left(\gamma_{j} \mid \mathcal{F}_{t_{j-1}}\right)\right|\right) \leq E\left(\left|\beta_{i}\right|\left|E\left(\gamma_{j} \mid \mathcal{F}_{t_{j-1}}\right)\right|\right) \text {. }
$$

According to the above identity,

$$
\left|E\left(\gamma_{j} \mid \mathcal{F}_{t_{j-1}}\right)\right| \leq\left|E\left(\left|\beta_{j}\right| \chi_{j} \mid \mathcal{F}_{t_{j-1}}\right)\right|+2 E\left(\left|\beta_{j}\right| I_{A_{j}^{c}} \mid \mathcal{F}_{t_{j-1}}\right) .
$$

Using Lemma 8.2 of the next section with $\eta_{u}=S_{t_{j}} / S_{t_{j-1}}-1, u=\left(\Delta t_{j}\right)^{1 / 2}$, we dominate the first term in the right-hand side by

$$
\kappa\left(S_{t_{i-1}}\left|\widehat{C}_{x t}\left(S_{t_{i-1}}, t_{i-1}\right)\right|+S_{t_{i-1}}^{3}\left|\widehat{C}_{x x x}\left(S_{t_{i-1}}, t_{i-1}\right)\right|\right)\left(\Delta t_{j}\right)^{3 / 2}
$$

It is easily seen from the explicit formulae that the coefficients above when $t_{j} \leq a$ can be dominated uniformly by $c_{a}\left(1+\sup _{t \leq 1} S_{t}\right)$, i.e. by a random variable having all moments. In the same range of indices we have also the bound $E\left(\beta_{i}^{2} \mid \mathcal{F}_{t_{i-1}}\right) \leq \zeta_{a}\left(\Delta t_{i}\right)^{2}$ where $\zeta_{a}$ a random variable having all moments. It follows from here that

$$
\sum_{i<j, t_{j} \leq a} E\left(\left|\beta_{i}\right|\left|E\left(\left|\beta_{j}\right| \chi_{j} \mid \mathcal{F}_{t_{j-1}}\right)\right|\right)=O\left(n^{-1 / 2}\right) .
$$

We estimate $P\left(A_{j}^{c} \mid \mathcal{F}_{t_{j-1}}\right)$ applying Lemma 8.3 of the next section with

$$
c_{1}\left(t_{j-1}\right):=\frac{S_{t_{j-1}}^{3} \widehat{C}_{x x x}\left(S_{t_{j-1}}, t_{j-1}\right)}{S_{t_{j-1}}^{2} \widehat{C}_{x x}\left(S_{t_{j-1}}, t_{j-1}\right)}, \quad c_{2}\left(t_{j-1}\right):=\frac{S_{t_{j-1}} \widehat{C}_{x t}\left(S_{t_{j-1}}, t_{j-1}\right)}{S_{t_{j-1}}^{2} \widehat{C}_{x x}\left(S_{t_{j-1}}, t_{j-1}\right)}
$$

and $c\left(t_{j-1}\right):=2\left(\left|c_{1}\left(t_{j-1}\right)\right|+\left|c_{2}\left(t_{j-1}\right)\right|+1\right)$. On the interval [0,a] the continuous process $c(t)$ can be dominated by a random variable $\xi_{a}$. Fix $\varepsilon>0$ and choose $N$ such that $P\left(\xi_{a}>N\right)<\varepsilon$. Lemma 8.3 implies that

$$
P\left(A_{j}^{c} \mid \mathcal{F}_{t_{j-1}}\right) \leq L_{N}\left(\Delta t_{j}\right)^{1 / 2} I_{\left\{c\left(t_{j-1}\right\}\right) \leq N}+I_{\left\{c\left(t_{j-1}\right)>N\right\}}
$$

and, therefore, $P\left(A_{j}^{c}\right) \leq L_{N}\left(\Delta t_{j}\right)^{1 / 2}+\varepsilon \leq 2 \varepsilon$ when $n$ is large enough. Using the Cauchy-Schwarz and Jensen inequalities we get that

$$
\begin{aligned}
\sum_{i<j, t_{j} \leq a} E\left(\left|\beta_{i}\right|\left|E\left(\left|\beta_{j}\right| I_{A_{j}^{c}} \mid \mathcal{F}_{t_{j-1}}\right)\right| \mid\right) & \leq \sum_{t_{i} \leq a}\left(E \beta_{i}^{2}\right)^{1 / 2} \sum_{t_{j} \leq a}\left(E \beta_{i}^{4}\right)^{1 / 4}\left(P\left(A_{j}^{c}\right)\right)^{1 / 4} \\
& \leq(2 \varepsilon)^{1 / 4} \sum_{t_{i} \leq a}\left(E \beta_{i}^{2}\right)^{1 / 2} \sum_{t_{j} \leq a}\left(E \beta_{j}^{4}\right)^{1 / 4}
\end{aligned}
$$

Both sums in the right-hand side are bounded because $E \beta_{j}^{2} \leq \kappa\left(\Delta t_{i}\right)^{2}$ and $E \beta_{j}^{4} \leq \kappa\left(\Delta t_{i}\right)^{4}$. By the choice of $\varepsilon$ the right-hand side can be made arbitrarily small. Thus, $n E\left(R_{1}^{24 n *}\right)^{2} \rightarrow 0$. 


\section{Asymptotics of Gaussian Integrals}

Let $\xi \in \mathcal{N}(0,1)$ and let $\eta_{u}:=e^{u \xi-\frac{1}{2} u^{2}}-1, u \in[0,1]$.

Lemma 8.1 The following asymptotical formulae holds as $u \rightarrow 0$ :

$$
\begin{aligned}
E\left[\eta_{u}^{2}-\eta_{-u}^{2}\right] I_{\left\{\eta_{u}>0\right\}} & =\frac{2}{\sqrt{2 \pi}} u^{3}+O\left(u^{4}\right), \\
E \eta_{u}^{2} \operatorname{sign} \eta_{u} & =\frac{2}{\sqrt{2 \pi}} u^{3}+O\left(u^{4}\right), \\
E \operatorname{sign} \eta_{u} & =-\frac{1}{\sqrt{2 \pi}} u+O\left(u^{3}\right) .
\end{aligned}
$$

Proof. Put

$$
Z(u):=\left(e^{u \xi-\frac{1}{2} u^{2}}-1\right)^{2}-\left(e^{-u \xi-\frac{1}{2} u^{2}}-1\right)^{2} .
$$

Then $Z(0)=Z^{\prime}(0)=Z^{\prime \prime}(0)=0, Z^{\prime \prime \prime}(0)=12\left(\xi^{3}-\xi\right)$, and the function $Z^{(4)}(u)$ is bounded by a random variable having moments of any order. Using the Taylor formula we obtain that

$$
E Z(u) I_{\left\{\xi \geq \frac{1}{2} u\right\}}=2 u^{3} E\left(\xi^{3}-\xi\right) I_{\left\{\xi \geq \frac{1}{2} u\right\}}+O\left(u^{4}\right), \quad u \rightarrow 0,
$$

and we obtain the first formula. The second formula is a corollary of the first one since

$$
E \eta_{u}^{2} \operatorname{sign} \eta_{u}=E Z(u) I_{\left\{\xi \geq \frac{1}{2} u\right\}}-E \eta_{u}^{2} I_{\left\{|\xi| \leq \frac{1}{2} u\right\}}
$$

and the last term is $O\left(u^{4}\right)$ as $u \rightarrow 0$. Finally,

$$
\begin{aligned}
E \operatorname{sign} \eta_{u}=P(\xi>u / 2)-P(\xi<u / 2) & =2(\Phi(0)-\Phi(u / 2)) \\
& =-\frac{1}{\sqrt{2 \pi}} u+\frac{1}{4} \varphi(\tilde{u}) \tilde{u} u^{2}
\end{aligned}
$$

where $\tilde{u} \in[0, u / 2]$.

Lemma 8.2 There exists a constant $\kappa$ such that for any $A \in \mathbf{R}$

$$
|E| \eta_{u}^{2}-A u^{2}\left|\operatorname{sign}\left(\eta_{u}^{2}-A u^{2}\right) \eta_{u}\right| \leq \kappa(1+|A|) u^{3} .
$$

Proof. Note that $|x| \operatorname{sign} x y=x \operatorname{sign} y$. Therefore the left-hand side of (8.1) is dominated by

$$
\left|E \eta_{u}^{2} \operatorname{sign} \eta_{u}\right|+|A| u^{2}\left|E \operatorname{sign} \eta_{u}\right|
$$

and the result holds by virtue of the previous lemma.

Lemma 8.3 For every $N>0$ there is a constant $L_{N}$ such that for all $u \in$ $[0,1]$

$$
P\left(\left|c_{1} \eta_{u}^{2}+c_{2} u^{2}\right|>\left|\eta_{u}\right|\right) \leq L_{N} I_{\{c \leq N\}} u+I_{\{c>N\}} .
$$

for any constants $c_{1}, c_{2}$ and $c:=2\left(\left|c_{1}\right|+\left|c_{2}\right|+1\right)$. 
Proof. Suppose that $N \geq c>2$, the only case where the work is needed. It is easy to see that

$$
\begin{aligned}
P\left(\left|c_{1} \eta_{u}^{2}+c_{2} u^{2}\right|>\left|\eta_{u}\right|\right) & \leq P\left((c / 2) \eta_{u}^{2}+(c / 2) u^{2}>\left|\eta_{u}\right|\right) \\
& \leq P\left(c\left|\eta_{u}\right|>1\right)+P\left(\left|\eta_{u}\right|<c u^{2}\right)
\end{aligned}
$$

The probabilities in the right-hand side as functions of $c$ are increasing and it remains to dominate their values at the point $c=N$. The required bound holds for the first probability in the right-hand side (and even with a constant which does not depend on $N$ ). Indeed, using the Chebyshev inequality, finite increments formula, and the bound $\varphi(x) \leq 1 / \sqrt{2 \pi}$ we have:

$$
P\left(N\left|\eta_{u}\right|>1\right) \leq \frac{1}{N} E\left|\eta_{u}\right| \leq \frac{1}{2} E\left|\eta_{u}\right|=\Phi(u / 2)-\Phi(-u / 2) \leq \frac{1}{\sqrt{2 \pi}} u .
$$

For $u \geq 1 / \sqrt{2 N}$ the second probability is dominated by linear functions with $L_{N} \geq \sqrt{2 N}$. For $u<1 / \sqrt{2 N}$ we write it as

$P\left(u / 2 \leq \xi<(1 / u) \ln \left(1+N u^{2}\right)+u / 2\right)+P\left((1 / u) \ln \left(1-N u^{2}\right)+u / 2<\xi<u / 2\right)$.

Using again the finite increments formula we obtain that

$$
P\left(u / 2 \leq \xi<(1 / u) \ln \left(1+N u^{2}\right)+u / 2\right) \leq \frac{1}{\sqrt{2 \pi}} N u
$$

On the interval $] 0,1 / \sqrt{2 N}$ [ we have the bound $(1 / u) \ln \left(1-N u^{2}\right) \geq-\kappa N u$ where $\kappa>0$ is the maximum of the function $-\ln (1-x) / x$ on the interval ] $0,1 / 2]$. It follows that

$$
P\left((1 / u) \ln \left(1-N u^{2}\right)+u / 2<\xi<u / 2\right) \leq \frac{1}{\sqrt{2 \pi}} \kappa N u .
$$

Thus, the second probability also admits a linear majorant on the whole interval $[0,1]$.

Acknowledgement. The authors express their thanks to anonymous referee for constructive criticism and helpful suggestions.

\section{References}

1. Geiss S. Quantitative approximation of certain stochastic integrals. Stochastics and Stochastic Reports, 73 (2002), 3-4, 241-270.

2. Granditz P., Schachinger W. Leland's approach to option pricing: The evolution of discontinuity. Mathematical Finance, 11 (2001), 3, 347-355.

3. Grannan E.R., Swindle G.H. Minimizing transaction costs of hedging strategies. Mathematical Finance, 6 (1996), 4, 341-364.

4. Denis E., Kabanov Yu.M. Functional limit theorem for Leland-Lott hedging strategy. To appear.

5. Kabanov Yu.M., Safarian M. On Leland's strategy of option pricing with transaction costs. Finance and Stochastics, 1, 3, 1997, 239-250.

6. Leland H. Option pricing and replication with transactions costs, Journal of Finance, XL (1985), 5, 1283-1301. 
7. Leland H. Comments on "Hedging errors with Leland's option model in the presence of transactions costs". Finance Research Letters, 4 (2007), 3, 200202.

8. Lott K. Ein Verfahren zur Replikation von Optionen unter Transaktionkosten in stetiger Zeit, Dissertation. Universität der Bundeswehr München. Institut für Mathematik und Datenverarbeitung, 1993.

9. Pergamenshchikov S. Limit theorem for Leland's strategy. The Annals of Applied Probability, 13 (2003), 1099-1118.

10. Schwartz L. Analyse Mathématique. Hermann, Paris, 1967.

11. Sekine J., Yano J. Hedging errors of Leland's strategies with timeinhomogeneous rebalancing. Preprint.

12. Zariphopoulou, T. Stochastic control methods in asset pricing. In: Handbook of Stochastic Analysis and Applications, Eds. Kannan D. and Lakshmikantham V. Marcel Dekker, New York-Basel, 2002.

13. Zhao Y., Ziemba W.T. Hedging errors with Leland's option model in the presence of transaction costs. Finance Research Letters, 4 (2007), 1, 49-58.

14. Zhao Y., Ziemba W.T. Comments on and corrigendum to "Hedging errors with Leland's option model in the presence of transaction costs". Finance Research Letters, 4 (2007), 3, 196-199. 